\title{
An algebraic formulation of kinematic isotropy and design of isotropic 6-6 Stewart platform manipulators
}

\author{
Sandipan Bandyopadhyay* and Ashitava Ghosal ${ }^{\dagger}$ \\ Department of Mechanical Engineering \\ Indian Institute of Science \\ Bangalore 560012
}

\begin{abstract}
In this paper, we present a new method to study and design isotropic spatial parallel manipulators. We construct the Jacobian matrices of linear and angular velocities symbolically and analyse them. The isotropy of the angular velocity and that of the linear velocity are treated in a decoupled manner using an algebraic formulation that lead to eigenproblems of certain symmetric matrices. The criteria for isotropy in the individual cases, as well as their combination are expressed in closed-form in terms of the minimum number of algebraic equations. The proposed method is applied to the design of isotropic 6-6 Stewart platforms having semi-regular hexagonal top and bottom platforms. Symbolic expressions for two different families of isotropic configurations are obtained. Several mechanically feasible configurations are presented demonstrating orientation, position, and combined kinematic isotropy. Methods are presented for designing a manipulator for combined isotropy with partially specified position and orientation, and for determining the isotropic configurations of a manipulator with a given geometry. The sensitivity of the isotropy conditions with respect to variation in the configuration parameters is studied numerically using the example of an existing manipulator.
\end{abstract}

\section{Introduction}

Dexterity of manipulation is one of the most important aspects of kinematic design of a manipulator. Various measures of dexterity have been proposed in the literature, which can be broadly categorised as local or at a given configuration, and global or over the entire workspace or subsets thereof. The local measures of dexterity involve the properties of the linear velocity Jacobian of the manipulator end-effector (denoted by $\boldsymbol{J}_{\boldsymbol{v}}$ ), and the properties of the associated velocity ellipsoid. Yoshikawa quantified the manipulability of a robotic mechanism as the volume of the velocity ellipsoid [1] defined as $\operatorname{det} \boldsymbol{J}_{\boldsymbol{v}} \boldsymbol{J}_{\boldsymbol{v}}^{T}$. Salisbury studied the sphericity of the velocity ellipsoid via the condition number, $\kappa_{2}\left(\boldsymbol{J}_{\boldsymbol{v}}\right) \triangleq \sigma_{\max }\left(\boldsymbol{J}_{\boldsymbol{v}}\right) / \sigma_{\min }\left(\boldsymbol{J}_{\boldsymbol{v}}\right)$, where $\sigma(\cdot)$ denotes one of the singular values of a matrix [2].

${ }^{*}$ The author is presently with the General Motors India Science Laboratory, Bangalore. e-mail: sandipan.bandyopadhyay@gm.com

${ }^{\dagger}$ Corresponding author. e-mail: asitava@mecheng.iisc.ernet.in 
The manipulator reaches optimal dexterity locally at a configuration if the condition number of its Jacobian matrix is unity at that configuration. A survey of the local measures of dexterity can be found in [3]. Early measures of global dexterity concentrate mostly on the size of the dexterous workspace defined to be the subset of the workspace that can be reached with all possible orientations $[4,5]$. Local measures based on the condition number have also been extended to global measures by integrating over a normalised volume of the workspace [6, 7]. More recently, Park and Brockett introduced a coordinate-free measure of global dexterity via the concept of kinematic distortion [8].

In this paper, we study the design of spatial parallel manipulators for optimal dexterity using the concept of local isotropy. For spatial manipulators, the orientation isotropy is analysed by considering the Jacobian matrix $\boldsymbol{J}_{\boldsymbol{\omega}}$ which maps the actuator rates to the angular velocity, and the position isotropy relates to the Jacobian matrix $\boldsymbol{J}_{\boldsymbol{v}}$ which maps the actuator rates to the linear velocity. The isotropy of the combined Jacobian, $\boldsymbol{J}=\left(\boldsymbol{J}_{\boldsymbol{\omega}} ; \boldsymbol{J}_{\boldsymbol{v}}\right)$, is termed as spatial isotropy [9]. Physically, these conditions imply that the manipulator end-effector can translate and/or rotate with equal ease in all spatial directions. This situation is extremally opposite to singularity, wherein the manipulator end-effector cannot translate and/or rotate in certain directions in spite of full actuation. In the reference [9] the authors point out that apart from optimizing dexterity, isotropy also leads to the minimum relative error in the velocity maps.

It is generally difficult to obtain the conditions of isotropy using the condition number $\kappa_{2}$ directly, as it requires the Jacobian matrix and its singular values in closed symbolic form. Due to the obvious computational difficulties, this approach has been applied only to the simplest of serial chains [12]. Researchers have also used the Frobenius norm (see, e.g., [10]) to calculate condition numbers without having to calculate the singular values first $[11]^{1}$. However, to the best of our knowledge, there are no reported application of this approach to parallel manipulators. Isotropy of parallel manipulators, in particular isotropy of Stewart platform manipulators (SPM's) has been studied numerically using the condition number criterion in reference [13]. In reference [14], the authors suggest a restructuring of the Jacobian matrix of a parallel manipulator, and identify its properties at an isotropic configuration. A similar formulation has been used to identify the spatially isotropic configurations of a Stewart platform [15]. Tsai and Huang study the isotropy conditions of the wrench transformation matrix $\boldsymbol{J}^{-T}$ in order to derive certain isotropy generators which lead to spatial isotropy [16].

The inherent kinematic constraints of a parallel manipulator, such as an SPM, introduce further difficulties in obtaining an isotropic configuration which is mechanically valid, i.e., which obeys the kinematic constraints of the manipulator, and is also mechanically feasible. It is possible to obtain isotropic configurations which are not mechanically feasible [14]. Recently, Tsai et al. have reported families of spatially isotropic configurations of SPM's, which are mechanically infeasible [16]. There seems to be no control over the architecture or configuration of the manipulator in their formulation, and in particular, it may be verified easily that the numerical results presented by them lead to manipulators whose top and and bottom platforms are not even convex polygons ${ }^{2}$. This fact, combined with awkwardly high base to height ratios (about $25: 1$ ), ruins the practical importance of these designs.

\footnotetext{
${ }^{1}$ In this paper, the condition number used follows the definition of $\kappa_{2}$.

${ }^{2}$ This is due to the fact that the isotropy generators, whose intersection with two different horizontal planes define the top and bottom platforms respectively, are not restricted to form the edges of a convex polyhedron. In fact, these are allowed to be nearly, or exactly horizontal.
} 
It is also possible to obtain a spatially isotropic configuration which is kinematically singular, and one such example may be found in reference [14]. Indeed, there are other examples in the literature, where the singularity aspect has been ignored, thereby leading to an infeasible set of isotropic configurations. Su et al. present numerically obtained optimal designs for the semi-regular Stewart platform manipulator (SRSPM), with geometrically similar top and bottom platforms [17]. As noted by several authors (see, for example $[18,19]$ ), such a manipulator is architecturally singular, i.e., it is singular over the entire task space, $S E(3)$, and therefore has little practical importance in the context of isotropy. A list of issues with the present state of literature in the area of isotropy can be found in reference [20].

Apart from the issues of kinematic constraints and singularities, there are other significant theoretical problems in studying isotropy of SPM's:

- The lack of symbolic expressions for the Jacobian matrices: For spatial parallel manipulators such as the SPM's, it is generally very difficult to obtain the angular and linear velocity Jacobian matrices. To bypass this problem, various alternative formulations for kinematic isotropy have been proposed, which do not need $\boldsymbol{J}_{\boldsymbol{\omega}}, \boldsymbol{J}_{\boldsymbol{v}}$ explicitly. However, such schemes are also inherently restricted to subsets of $S E(3)$, and fail to explore the entire task space. For example, in [15], two matrices $\boldsymbol{A}, \boldsymbol{B}$ multiply the output and the input rates of a Stewart platform manipulator respectively, and when they are both isotropic the manipulator is said to be in an isotropic configuration. However, as we show later in this paper, isotropy of $\boldsymbol{B}$ eliminates the possibility of obtaining a configuration showing combined isotropy ${ }^{3}$.

- The lack of a natural length scale of $S E(3)$ : Even when the Jacobian matrices are available symbolically, their use in isotropy analysis is complicated due to the fact that the Jacobian elements are not dimensionally homogeneous - the entries corresponding to the angular and linear velocity differ by a length scale. Therefore isotropy of the Jacobian $\boldsymbol{J}$ can change by a simple change of the units. Researchers have tried to circumvent this problem using length scales (see, for example $[21,14]$ ) relevant to a given problem. However, in theory, the group of rigid-body motions, $S E(3)$, does not admit any natural length scale [22, 23], and therefore the choice of such a scale, $L_{s}$, can never be unique, and/or globally applicable.

The length scales used in literature can be classified in two groups: (a) intrinsic, and (b) extrinsic. The scales used in $[14,15]$ fall in category (a), and they are defined such that if $\boldsymbol{J}_{\boldsymbol{\omega}}$, $\boldsymbol{J}_{\boldsymbol{v}}$ were isotropic individually, their singular values would attain the same numerical value. In particular, in such cases, the final expression of $L_{s}$ would reduce to $L_{s}=\frac{\sigma\left(\boldsymbol{J}_{\boldsymbol{v}}\right)}{\sigma\left(\boldsymbol{J}_{\boldsymbol{\omega}}\right)}$, i.e., the scale itself is a function of the final solution. In category (b), the scale is suggested by the designer explicitly, and it is not necessarily a function of the elements of $\boldsymbol{J}_{\boldsymbol{\omega}}$ and $\boldsymbol{J}_{\boldsymbol{v}}$. The choice of such a scale is motivated by the geometry of the problem, and can affect the end results obtained. Pittens and Podhorodeski [13] explain the significance of such scales, and use the radius of the top platform of an SRSPM as $L_{s}$ to study its isotropy.

To overcome the difficulties mentioned above, we study the individual Jacobian matrices, $\boldsymbol{J}_{\boldsymbol{\omega}}$, and $\boldsymbol{J}_{\boldsymbol{v}}$ for their isotropy. We also define a combined isotropy where the matrices $\boldsymbol{J}_{\boldsymbol{\omega}}$ and $\boldsymbol{J}_{\boldsymbol{v}}$ are isotropic individually. However, their singular values are not necessarily equal. The notion of

\footnotetext{
${ }^{3}$ The isotropy of $\boldsymbol{B}$ requires that the all the legs of the manipulator have equal length. We shall see in section 4.1 this is quite restrictive.
} 
combined isotropy avoids the problems of length scale and is a subset of spatial isotropy. We use symbolic computations extensively to obtain the $\boldsymbol{J}_{\boldsymbol{\omega}}$ and $\boldsymbol{J}_{\boldsymbol{v}}$ matrices in closed-form, by inverting the wrench transformation matrix for the top platform. The formulation can be applied to study isotropy of manipulators of any architecture, whose forward velocity maps are available in terms of the actuator rates alone.

In this paper, we apply our formulation to study the isotropy of the semi-regular Stewart platform manipulator. The motivation of studying this particular class of SPM's derives mainly from its wide-spread usage in the industry, such as in commercial flight simulators (e.g., CAE 500 Seri), parallel machine tools [24], attitude fine tuning platform for large radio telescopes [17] (see [25] for an interesting list of applications of this manipulator in industry, research, and entertainment). We identify two families of isotropic manipulators, the first of which encompasses those reported in $[14,15]$. We show that combined isotropy of $\boldsymbol{J}_{\boldsymbol{\omega}}, \boldsymbol{J}_{\boldsymbol{v}}$ cannot occur within this family, and study a more generalised family of configurations. In this case, we obtain closed-form solutions for isotropy in orientation, position, and combined sense. We show symbolically that no kinematically feasible configuration exists within this family which demonstrates spatial isotropy. We also present methods to obtain combined isotropy in an existing SRSPM, and to design an SRSPM for partially specified position and orientation. The theory developed in this paper is illustrated by several examples.

The organisation of the paper is as follows: in section 2, we discuss the formulations for distribution of linear and angular velocities, and derive the conditions for position, orientation, spatial and combined isotropy. In section 3, we present the conditions for isotropy of an SRSPM in terms of closed-form algebraic equations. In section 4, we present two sets of closed-form solutions to these equations, leading to corresponding families of isotropic configurations. In the next section, we present the methods for obtaining the combined isotropy configurations of an SRSPM of given geometry, and also the design process for combined isotropy at partially specified position and orientation. In section 6 , we study numerically the sensitivity of the isotropy conditions to the variations in configuration parameters around the isotropic configurations. Finally, we present the conclusions and discuss the scope of future work in section 7 .

\section{Isotropy conditions of a manipulator}

In this section, we derive the isotropy conditions of a general manipulator from its forward velocity maps. First, we describe the mathematical formulation for analysing the distribution of angular and linear velocities of the manipulator. We follow the differential-geometric approach presented in [26], and reduce the problem of velocity distribution (instantaneously at a given configuration) to eigenproblems of symmetric matrices. The conditions for linear and angular isotropy are then derived in terms of algebraic equations involving the coefficients of the characteristic polynomials associated with the above eigenproblems.

\subsection{Formulation of the velocity distribution and isotropy}

We assume in the following that the angular velocity $\boldsymbol{\omega}$ and the linear velocity $\boldsymbol{v}_{\boldsymbol{p}}$ are available via linear maps of the actuated joint rates $\dot{\boldsymbol{\theta}} \in \mathbb{R}^{n}$ alone, where $n$ is the degree-of-freedom of the manipulator. In the context of parallel and hybrid manipulators, this means that the passive joint rates have been eliminated in obtaining the velocity Jacobian matrices, and we can write $\boldsymbol{\omega}$ and $\boldsymbol{v}_{\boldsymbol{p}}$ 
in terms of the respective equivalent Jacobian matrices as

$$
\begin{aligned}
\boldsymbol{\omega} & =\boldsymbol{J}_{\boldsymbol{\omega}} \dot{\boldsymbol{\theta}} \\
\boldsymbol{v}_{\boldsymbol{p}} & =\boldsymbol{J}_{\boldsymbol{v}} \dot{\boldsymbol{\theta}}
\end{aligned}
$$

The extremal values of the linear and angular velocity, subject to a constraint $\|\dot{\boldsymbol{\theta}}\|=1$, can be obtained from the two eigenproblems below:

$$
\begin{aligned}
\boldsymbol{g} \dot{\boldsymbol{\theta}} & =\lambda \dot{\boldsymbol{\theta}} \\
\boldsymbol{g}_{\boldsymbol{v}} \dot{\boldsymbol{\theta}} & =\lambda_{v} \dot{\boldsymbol{\theta}}
\end{aligned}
$$

where $\boldsymbol{g}=\boldsymbol{J}_{\boldsymbol{\omega}}^{T} \boldsymbol{J}_{\boldsymbol{\omega}}$ and $\boldsymbol{g}_{\boldsymbol{v}}=\boldsymbol{J}_{\boldsymbol{v}}^{T} \boldsymbol{J}_{\boldsymbol{v}}$. It is obvious from equations $(2,3)$ that $\lambda=\sigma^{2}\left(\boldsymbol{J}_{\boldsymbol{\omega}}\right)$ and $\lambda_{v}=\sigma^{2}\left(\boldsymbol{J}_{\boldsymbol{v}}\right)$. The eigenvalues also signify the squares of the extremal values of $\|\boldsymbol{\omega}\|$ and $\|\boldsymbol{v}\|$ respectively under the constraint $\|\dot{\boldsymbol{\theta}}\|=1$ :

$$
\begin{aligned}
\lambda & =\|\boldsymbol{\omega}\|_{*}^{2} \\
\lambda_{v} & =\|\boldsymbol{v}\|_{*}^{2}
\end{aligned}
$$

where $\|\boldsymbol{\omega}\|_{*},\|\boldsymbol{v}\|_{*}$ equal the half of the three principal axes of the ellipsoids of angular, and linear velocity respectively [26]. Clearly, the eigenvalues $\lambda, \lambda_{v}$ are nonnegative. However, at the most three of the eigenvalues are nonzero in each case, as the rank of $\boldsymbol{J}_{\boldsymbol{\omega}}$ or $\boldsymbol{J}_{\boldsymbol{v}}$ cannot exceed three. If $\operatorname{dim}(\boldsymbol{g})=n$ with $n>3$, at least $(n-3)$ eigenvalues of $\boldsymbol{g}$ are zeros, and we can write the characteristic equation of $\boldsymbol{g}$ with real coefficients $a_{i}$ as:

$$
0= \begin{cases}\lambda^{2}+a_{1} \lambda+a_{2} & n=2 \\ \lambda^{3}+a_{1} \lambda^{2}+a_{2} \lambda+a_{3} & n=3 \\ \lambda^{n-3}\left(\lambda^{3}+a_{1} \lambda^{2}+a_{2} \lambda+a_{3}\right) & n>3\end{cases}
$$

The characteristic equation of $\boldsymbol{g}_{\boldsymbol{v}}$ has exactly the same form as above. However, in order to distinguish it from equation (4), we use the notations $b_{i}$ for the coefficients, and $\lambda_{v}$ for the eigenvalues for this case.

Identification of the extremal values of $\|\boldsymbol{\omega}\|$ and $\|\boldsymbol{v}\|$ leads to a natural description of isotropy. When the three $\lambda_{v}$ 's are equal and nonzero, the linear velocity ellipsoid [26] reduces to a sphere and we get the condition for the isotropy of linear velocity. Similarly, isotropy of angular velocities would require that the eigenvalues $\lambda$ are all equal. This implies that the nontrivial roots of equation (4) should be equal, and not all of $a_{1}, a_{2}, a_{3}$ can be zero.

It may be noted here that the coefficients $a_{i}, b_{i}$ can be computed in closed-form as described in Appendix A. The nontrivial roots of equation (4) can also be obtained explicitly in terms of $a_{i}$ using Sridhar Acharya's and Cardan's formulæ for the quadratic and cubic cases respectively [27]. However, it is not required to compute the roots explicitly in order to obtain the conditions for isotropy from their equality. Instead, those conditions can be easily formed as algebraic equations in the coefficients $a_{i}$ etc. as follows.

For the case $n=2$, we equate the discriminant to zero and obtain the following condition:

$$
a_{1}^{2}-4 a_{2}=0
$$


For the case $n \geq 3$, we consider the nontrivial cubic part of equation (4):

$$
\lambda^{3}+a_{1} \lambda^{2}+a_{2} \lambda+a_{3}=0
$$

Using the standard transformation

$$
\lambda=z-\frac{a_{1}}{3}
$$

the cubic is reduced to its standard form:

$$
z^{3}+P z+Q=0
$$

where

$$
P=\frac{3 a_{2}-a_{1}^{2}}{3}, \quad Q=\frac{2 a_{1}^{3}-9 a_{1} a_{2}+27 a_{3}}{27}
$$

Clearly if $P=Q=0$, then $z=0$. Using equation (7) the roots of equation (6) are obtained as:

$$
\lambda_{i}=-\frac{a_{1}}{3}, \quad i=1,2,3
$$

Therefore, the conditions for the cubic to have all roots equal are

$$
\begin{aligned}
& 3 a_{2}-a_{1}^{2}=0 \\
& 2 a_{1}^{3}-9 a_{1} a_{2}+27 a_{3}=0
\end{aligned}
$$

For the purpose of symbolic computations, the second condition in equation (12) can be simplified using the first in equation (11) to arrive at an equivalent set of conditions:

$$
\begin{aligned}
& 3 a_{2}-a_{1}^{2}=0 \\
& 27 a_{3}-a_{1}^{3}=0
\end{aligned}
$$

The conditions for equal $\lambda_{v}$ isotropy can be obtained in the same fashion. In the following, we list down the conditions for the different types of isotropy considered in this paper.

\subsection{Conditions for different types of isotropy}

We consider three cases of isotropy in the following:

A. Orientation ( $\boldsymbol{\omega}$-isotropy): $\boldsymbol{J}_{\boldsymbol{\omega}}$ alone is isotropic.

B. Position ( $\boldsymbol{v}$-isotropy): $\boldsymbol{J}_{\boldsymbol{v}}$ alone is isotropic.

C. Combined: Both $\boldsymbol{J}_{\boldsymbol{\omega}}, \boldsymbol{J}_{\boldsymbol{v}}$ are isotropic.

It may be mentioned that the case $\mathrm{C}$ above along with the additional condition $\boldsymbol{J}_{\boldsymbol{\omega}}^{T} \boldsymbol{J}_{\boldsymbol{v}}=\mathbf{0}$ has been termed as spatial isotropy [9]. We use the term combined to emphasise the difference of our results from others. We also show in section 4.2.4 that this additional condition cannot be satisfied in a non-singular class of configurations of the SRSPM showing combined isotropy. 


\section{Case A: Conditions for $\omega$-isotropy}

As described above, the conditions in this case are:

$$
a_{1}^{2}-4 a_{2}=0, \quad n=2
$$

and for $n \geq 3$ :

$$
\begin{aligned}
& 3 a_{2}-a_{1}^{2}=0 \\
& 27 a_{3}-a_{1}^{3}=0
\end{aligned}
$$

\section{Case B: Conditions for $v$-isotropy}

Following the derivation of $\boldsymbol{\omega}$-isotropy conditions above, the conditions for $\boldsymbol{v}$-isotropy may be written as:

$$
b_{1}^{2}-4 b_{2}=0, \quad n=2
$$

and for $n \geq 3$ :

$$
\begin{aligned}
& 3 b_{2}-b_{1}^{2}=0 \\
& 27 b_{3}-b_{1}^{3}=0
\end{aligned}
$$

\section{Case C: Conditions for combined isotropy}

In this case, we have $\boldsymbol{\omega}$-isotropy and $\boldsymbol{v}$-isotropy simultaneously. The conditions that apply in this case are simply the union of the conditions in cases A and B. We reproduce them below for the sake of explicit reference:

For $n=2$,

$$
\begin{aligned}
& a_{1}^{2}-4 a_{2}=0 \\
& b_{1}^{2}-4 b_{2}=0
\end{aligned}
$$

and for $n \geq 3$ :

$$
\begin{aligned}
& 3 a_{2}-a_{1}^{2}=0 \\
& 27 a_{3}-a_{1}^{3}=0 \\
& 3 b_{2}-b_{1}^{2}=0 \\
& 27 b_{3}-b_{1}^{3}=0
\end{aligned}
$$

\section{Isotropy conditions of an SRSPM}

In this section, we apply the theory developed in section 2 to formulate the isotropy conditions of an SRSPM. In addition to its wide-spread technical applications mentioned earlier, the other motivations to choose this manipulator as our example are: (a) it is probably the most well-studied spatial parallel manipulator (see section 1 for some of the references) (b) no feasible configuration of any Stewart platform manipulator demonstrating combined isotropy is reported in literature to the best of our knowledge. 


\subsection{Description of the manipulator geometry and kinematic constraints}

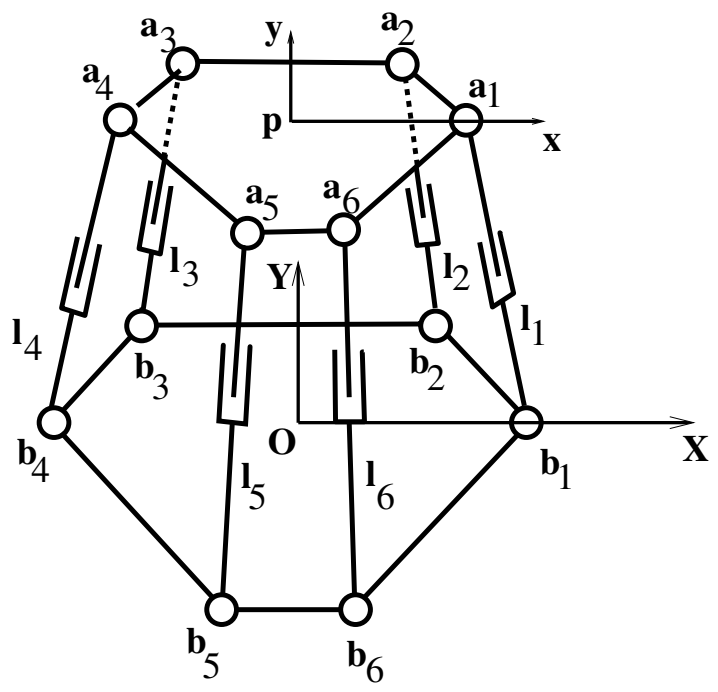

(a) The manipulator

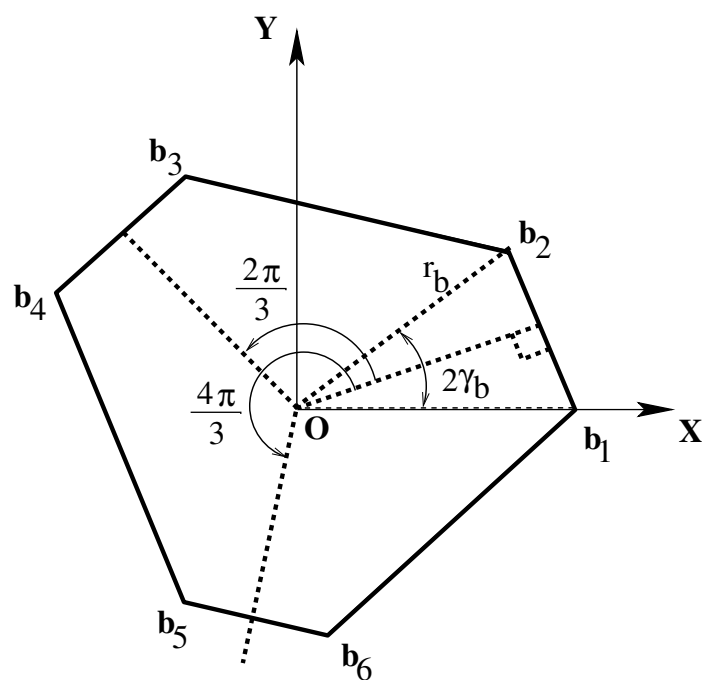

(b) Bottom platform

Figure 1: Geometry of the Stewart platform manipulator

The manipulator along with the frames of reference used is shown in figure 1(a). The bottom platform, shown in figure 1(b), has the legs arranged in a circle, with each pair lying symmetrically on either side of three axes of symmetry in the plane. The axes are $\frac{2 \pi}{3}$ apart from each other, while the adjacent pair of legs have an angular spacing $2 \gamma_{b}$. Without any loss of generality, we scale the radius of the circumcircle of the bottom platform, $r_{b}$, to unity, thus eliminating one parameter from all subsequent analysis, and rendering all other length parameters used in this paper dimensionless. The top platform geometry is similar, except that the radius of its circumcircle is $r_{t}$, and a leg spacing $2 \gamma_{t}$.

The kinematic constraints defining the manipulator are written in the task-space variables. The center of the top platform is described in the base frame as $\boldsymbol{p}=(x, y, z)^{T}$. The top platform orientation is described by the matrix $\boldsymbol{R} \in S O(3)$, where $\boldsymbol{R}=\boldsymbol{R}_{z}(\phi) \boldsymbol{R}_{x}\left(\theta_{x}\right) \boldsymbol{R}_{y}\left(\theta_{y}\right)^{4}$. The loopclosure equations are written as

$$
\boldsymbol{p}+\boldsymbol{R} \boldsymbol{a}_{i}-\boldsymbol{b}_{i}-l_{i} \boldsymbol{s}_{i}=\mathbf{0}, \quad i=1, \ldots, 6
$$

where $l_{i}$ denotes the length of the $i$ th leg and $\boldsymbol{a}_{i}, \boldsymbol{b}_{i}$ locate the leg connection points with respect to the platform centers in respective frames (see figure 1(a)), and $s_{i}$ denotes the $i$ th screw axis along the respective leg.

\subsection{Formulation of the velocity Jacobian matrices}

Differentiating equation (23) with respect to time, we obtain

$$
\boldsymbol{v}_{\boldsymbol{p}}+\dot{\boldsymbol{R}} \boldsymbol{a}_{i}-i_{i} \boldsymbol{s}_{i}-l_{i} \dot{\boldsymbol{s}}_{i}=\mathbf{0}, \quad i=1, \ldots, 6
$$

\footnotetext{
${ }^{4}$ In this paper, we denote the rotation about the $X$ axis through an angle $\theta$ as $\boldsymbol{R}_{x}(\theta)$ etc.
} 
where $\boldsymbol{v}_{\boldsymbol{p}}=\dot{\boldsymbol{p}}$. Note that $\boldsymbol{s}_{i}$ is a unit vector, therefore $\dot{\boldsymbol{s}}_{i}=\boldsymbol{\omega}_{i} \times \boldsymbol{s}_{i}$, where $\boldsymbol{\omega}_{i}$ is the space-fixed angular velocity of the $i$ th leg. Also note that $\dot{\boldsymbol{R}} \boldsymbol{a}_{i}$ can be written as

$$
\dot{\boldsymbol{R}}\left(\boldsymbol{R}^{T} \boldsymbol{R}\right) \boldsymbol{a}_{i}=\left(\dot{\boldsymbol{R}} \boldsymbol{R}^{T}\right)\left(\boldsymbol{R} \boldsymbol{a}_{i}\right)=\boldsymbol{\omega}_{p} \times\left(\boldsymbol{R} \boldsymbol{a}_{i}\right)
$$

where $\boldsymbol{\omega}_{\boldsymbol{p}}$ is the space-fixed angular velocity of the top platform [22]. We rewrite equation (24) as

$$
\boldsymbol{v}_{\boldsymbol{p}}+\boldsymbol{\omega}_{p} \times\left(\boldsymbol{R} \boldsymbol{a}_{i}\right)-i_{i} \boldsymbol{s}_{i}-l_{i} \boldsymbol{\omega}_{i} \times \boldsymbol{s}_{i}=\mathbf{0}, \quad i=1, \ldots, 6
$$

Equation (25) involves both active and passive (unactuated) joint variables, and the passive variables appear explicitly in $s_{i}$ alone. Therefore, we take a dot product of the above equation with $\boldsymbol{s}_{i}$ to eliminate all passive variables, and obtain:

$$
\boldsymbol{s}_{i} \cdot \boldsymbol{v}_{\boldsymbol{p}}+\boldsymbol{s}_{i} \cdot \boldsymbol{\omega}_{p} \times\left(\boldsymbol{R} \boldsymbol{a}_{i}\right)=i_{i}, \quad i=1, \ldots, 6
$$

Substituting for $\boldsymbol{s}_{i}$ from equation (23), equation (26) can be cast in the matrix form as

$$
\left(\begin{array}{cc}
\frac{1}{l_{1}}\left(\left(\boldsymbol{R} \boldsymbol{a}_{1}\right) \times\left(\boldsymbol{p}-\boldsymbol{b}_{1}\right)\right)^{T} & \frac{1}{l_{1}}\left(\boldsymbol{p}+\boldsymbol{R} \boldsymbol{a}_{1}-\boldsymbol{b}_{1}\right)^{T} \\
\frac{1}{l_{2}}\left(\left(\boldsymbol{R} \boldsymbol{a}_{2}\right) \times\left(\boldsymbol{p}-\boldsymbol{b}_{2}\right)\right)^{T} & \frac{1}{l_{2}}\left(\boldsymbol{p}+\boldsymbol{R} \boldsymbol{a}_{2}-\boldsymbol{b}_{2}\right)^{T} \\
\frac{1}{l_{3}}\left(\left(\boldsymbol{R} \boldsymbol{a}_{3}\right) \times\left(\boldsymbol{p}-\boldsymbol{b}_{3}\right)\right)^{T} & \frac{1}{l_{3}}\left(\boldsymbol{p}+\boldsymbol{R} \boldsymbol{a}_{3}-\boldsymbol{b}_{3}\right)^{T} \\
\frac{1}{l_{4}}\left(\left(\boldsymbol{R} \boldsymbol{a}_{4}\right) \times\left(\boldsymbol{p}-\boldsymbol{b}_{4}\right)\right)^{T} & \frac{1}{l_{4}}\left(\boldsymbol{p}+\boldsymbol{R} \boldsymbol{a}_{4}-\boldsymbol{b}_{4}\right)^{T} \\
\frac{1}{l_{5}}\left(\left(\boldsymbol{R} \boldsymbol{a}_{5}\right) \times\left(\boldsymbol{p}-\boldsymbol{b}_{5}\right)\right)^{T} & \frac{1}{l_{5}}\left(\boldsymbol{p}+\boldsymbol{R} \boldsymbol{a}_{5}-\boldsymbol{b}_{5}\right)^{T} \\
\frac{1}{l_{6}}\left(\left(\boldsymbol{R} \boldsymbol{a}_{6}\right) \times\left(\boldsymbol{p}-\boldsymbol{b}_{6}\right)\right)^{T} & \frac{1}{l_{6}}\left(\boldsymbol{p}+\boldsymbol{R} \boldsymbol{a}_{6}-\boldsymbol{b}_{6}\right)^{T}
\end{array}\right)\left(\begin{array}{c}
\boldsymbol{\omega}_{p} \\
\boldsymbol{v}_{\boldsymbol{p}}
\end{array}\right)=\left(\begin{array}{c}
i_{1} \\
i_{2} \\
i_{3} \\
i_{4} \\
i_{5} \\
i_{6}
\end{array}\right)
$$

The $6 \times 6$ matrix on the left-hand side of equation (27) may be easily recognised as the transpose of the wrench transformation matrix for the top platform [19]. Denoting it by $\boldsymbol{H}^{T}$, we can express the linear and angular velocities as

$$
\begin{aligned}
\left(\begin{array}{c}
\boldsymbol{\omega}_{p} \\
\boldsymbol{v}_{\boldsymbol{p}}
\end{array}\right) & =\left(\boldsymbol{H}^{T}\right)^{-1} \boldsymbol{i} \\
& =\left(\begin{array}{c}
\boldsymbol{J}_{\boldsymbol{\omega}} \\
\boldsymbol{J}_{\boldsymbol{v}}
\end{array}\right) \boldsymbol{l}, \quad \operatorname{det} \boldsymbol{H} \neq 0
\end{aligned}
$$

The symbolic expressions for the Jacobian matrices $\boldsymbol{J}_{\boldsymbol{\omega}}$ and $\boldsymbol{J}_{\boldsymbol{v}}$ can be obtained from equation (28) when $\boldsymbol{H}$ is non-singular. Note that this step involves the symbolic inversion of a $6 \times 6$ matrix with complicated (trigonometric and algebraic) multi-term entries. This requires extensive symbolic computations on a computer algebra system (CAS). The computational complexity involved is a well-known problem in the design and analysis of spatial parallel manipulators such as the SPM [25], and closed-form expressions for the velocity Jacobian matrices are not available in literature to the best of our knowledge. In this work, however, we perform the inversion explicitly using the computer algebra system Mathematica [28]. It turns out that the built-in matrix inversion command Inverse is unable to deliver the result for the present problem in reasonable time. However, using the concept of classical adjoint of a matrix [10], we can obtain the inverse explicitly as

$$
\left(\boldsymbol{H}^{T}\right)^{-1}=\frac{\operatorname{adj} \boldsymbol{H}^{T}}{\operatorname{det} \boldsymbol{H}^{T}}
$$


Attempts to obtain explicit expressions for $\boldsymbol{J}_{\boldsymbol{\omega}}$ and $\boldsymbol{J}_{\boldsymbol{v}}$ directly from equation (29), results in memory overflow (which is $2 \mathrm{~GB}$ in our case). To circumvent this problem, we replace the actual determinant of $\boldsymbol{H}$ by the symbol $D_{\boldsymbol{H}}$, and scale the Jacobian matrices to obtain

$$
\left(\begin{array}{c}
\boldsymbol{J}_{\boldsymbol{\omega}}^{s} \\
(3 \times 6) \\
--- \\
\boldsymbol{J}_{\boldsymbol{v}}^{s} \\
(3 \times 6)
\end{array}\right)=\frac{\operatorname{adj}\left(\boldsymbol{H}^{T}\right)}{D_{\boldsymbol{H}}}
$$

such that $\boldsymbol{J}_{\boldsymbol{\omega}}^{s}=D_{\boldsymbol{H}} \boldsymbol{J}_{\boldsymbol{\omega}}$ and $\boldsymbol{J}_{\boldsymbol{v}}^{s}=D_{\boldsymbol{H}} \boldsymbol{J}_{\boldsymbol{v}}$. The isotropy conditions in equation (22) are homogeneous in the elements of $\boldsymbol{g}$ and $\boldsymbol{g}_{\boldsymbol{v}}$, and therefore they remain unchanged if we scale $\boldsymbol{J}_{\boldsymbol{\omega}}, \boldsymbol{J}_{\boldsymbol{v}}$ by the quantity $\frac{1}{D_{\boldsymbol{H}}}$ as long as $D_{\boldsymbol{H}} \neq 0$. In other words, we can use $\boldsymbol{g}^{s}=\boldsymbol{J}_{\boldsymbol{\omega}}^{s T} \boldsymbol{J}_{\boldsymbol{\omega}}^{s}$ and $\boldsymbol{g}_{\boldsymbol{v}}^{s}=\boldsymbol{J}_{\boldsymbol{v}}^{s T} \boldsymbol{J}_{\boldsymbol{v}}^{s}$ instead of $\boldsymbol{g}$ and $\boldsymbol{g}_{\boldsymbol{v}}$ respectively for the analysis of isotropy. In this process, a lot of memory space and computer time is saved by avoiding the division by the actual determinant in equation (29) and further symbolic computations are made possible.

\subsection{Formulation of the isotropy conditions}

Once the Jacobian matrices $\boldsymbol{J}_{\boldsymbol{\omega}}^{s}$ and $\boldsymbol{J}_{\boldsymbol{v}}^{s}$ are formed explicitly, formulation of the isotropy conditions can be done easily following section 2.2 . We summarise below the computational steps involved for all the cases $\mathrm{A}, \mathrm{B}$, and $\mathrm{C}$ of section 2.2:

1. Form the symmetric matrix $\boldsymbol{g}^{s}=\boldsymbol{J}_{\boldsymbol{\omega}}^{s T} \boldsymbol{J}_{\boldsymbol{\omega}}^{s}$

2. Form the symmetric matrix $\boldsymbol{g}_{\boldsymbol{v}}^{s}=\boldsymbol{J}_{\boldsymbol{v}}^{s T} \boldsymbol{J}_{\boldsymbol{v}}^{s}$

3. Compute the coefficients of the characteristic equations of $\boldsymbol{g}^{s}, \boldsymbol{g}_{\boldsymbol{v}}^{s}$ using equation (62) (see Appendix A).

4. Use equations $(21,22)$ or any subset of the same, as appropriate for the different cases of isotropy.

\section{Closed-form results on the isotropy of an SRSPM}

We now describe some closed-form results for the different cases of isotropy of the SRSPM using the formulation developed in the last section. The isotropy equations constructed therein allow us to solve the problems of analysis and synthesis within the same setup, in addition to studying the isotropic configurations in general. In this context, by analysis we mean obtaining the isotropic configurations of a manipulator with a given architecture, and by synthesis, the determination of the architectural parameters such that the manipulator is isotropic in a given configuration.

The independent variables involved in the isotropy equations are the position of the top platform $\boldsymbol{p}=(x, y, z)^{T}$, the orientation variables $\alpha, \beta, \phi$, and the architectural variables $r_{t}, \gamma_{b}$ and $\gamma_{t}$. The natural restrictions on the architectural parameters for mechanically feasible design would be the following:

- $\overline{r_{t}} \geq r_{t} \geq \underline{r_{t}}$ where $\overline{r_{t}}, \underline{r_{t}}>0$ are two prescribed limits. We adopt in this work $\overline{r_{t}}=1, \underline{r_{t}}=1 / 4$. 
- $\pi / 3 \geq \gamma_{b}, \gamma_{t} \geq 0$. At both ends of these limits, the hexagonal platforms reduce to triangles, and beyond these limits the leg connection points with the platforms cross over, and the legs can interfere mutually.

- The moving platform is above the fixed one, i.e., $z>0$.

- $\gamma_{b} \neq \gamma_{t}$. If the platforms are scaled versions of each other, the manipulator becomes architecturally singular $[18,19]$.

Any solution for the architecture within these restrictions would be termed as feasible or valid. Other mechanical constraints, such as joint limits, leg limits, and physical dimensions of the legs etc. are not considered in the present work. As a result, we do not impose any ranges on the values of the position and orientation variables, except $z>0$. We start with the following assumptions which enable us to perform symbolic computations and obtain algebraic expressions:

- Isotropic configurations and corresponding architectures are obtained only for the case when the manipulator is in its home configuration. The home configuration is defined as $x=y=0$, $\alpha=\beta=0$. In other words, displacement along and rotation about only the $Z$ axis is considered.

- The leg lengths have special relationships among themselves. We consider two families of isotropic configurations: family 1 has all leg lengths equal, while family 2 has alternate leg lengths equal.

These restrictions by no means reflect any limitation of our formulation; relaxing these has only the effect of increasing the complexity of the problem ${ }^{5}$. Note also that in the literature $[9,21,15,16]$ the only configuration studied is a subset of family 1, i.e., all leg lengths are equal. We discuss both the families in detail below.

\subsection{Isotropic configurations: family 1}

The restrictions defining family 1 are:

$$
x=y=0, \alpha=\beta=0, l_{i}=L>0, i=1, \ldots, 6
$$

The determinant of $\boldsymbol{H}$ in this case is given by

$$
D_{\boldsymbol{H}}=\frac{54 r_{t}^{3} z^{3} \cos \left(\gamma_{b}-\gamma_{t}-\phi\right) \sin \left(\gamma_{b}-\gamma_{t}\right)}{L^{6}}
$$

Therefore the matrix $\boldsymbol{H}$ is singular when one or more of the following conditions hold:

1. The top platform radius $r_{t}$ is zero.

2. The vertical displacement of $\boldsymbol{p}$ is zero, i.e., the top and bottom platform centers coincide.

\footnotetext{
${ }^{5}$ Although we do not have a proof, we have not been able to find any other family of isotropic configuration (namely with all unequal leg lengths or at $x, y, \alpha, \beta \neq 0$ ) for the SRSPM's studied by us. This is in spite of extensive searches using various methods.
} 
3. Top platform rotation is given by $\phi=\gamma_{b}-\gamma_{t} \pm \pi / 2$. This is a singular configuration of the SPM as reported by Fichter [29], and verified by others [30, 19].

4. The geometry of the top platform is a scaled version of the bottom platform, i.e., $\gamma_{b}=\gamma_{t}$. Under this condition, the manipulator becomes architecturally singular [18, 19], i.e., it is singular over the entire task-space.

The leg lengths $l_{i}$ can be obtained from the equation (23) as

$$
L^{2}=l_{i}^{2}=\left(\boldsymbol{p}+\boldsymbol{R} \boldsymbol{a}_{i}-\boldsymbol{b}_{i}\right)^{T}\left(\boldsymbol{p}+\boldsymbol{R} \boldsymbol{a}_{i}-\boldsymbol{b}_{i}\right), \quad i=1, \ldots, 6
$$

In the present case, all the lengths are identical, and this results in only the following two distinct equations among the six above:

$$
\begin{aligned}
& L^{2}=1+r_{t}^{2}+z^{2}-2 r_{t} \cos \phi \\
& L^{2}=1+r_{t}^{2}+z^{2}-2 r_{t} \cos \left(2 \gamma_{b}-2 \gamma_{t}-\phi\right)
\end{aligned}
$$

Eliminating $L$ between the above two equations, we obtain the single equation defining the kinematic constraints as

$$
r_{t} \sin \left(\gamma_{b}-\gamma_{t}\right) \sin \left(\gamma_{b}-\gamma_{t}-\phi\right)=0
$$

Since $r_{t} \sin \left(\gamma_{b}-\gamma_{t}\right)=0$ implies singularity as seen above, we have the kinematic constraint as

$$
\phi=\gamma_{b}-\gamma_{t}, \gamma_{b}-\gamma_{t}+\pi \quad \phi \in[0,2 \pi]
$$

The two solutions of $\phi$ define two cases of solutions within family 1 .

\subsubsection{Case 1: $\phi=\gamma_{b}-\gamma_{t}$}

$\boldsymbol{\omega}$-isotropy - In this case, the symmetry of the problem forces two of the $\lambda$ 's to be identical in equation (4). As a consequence, the $\boldsymbol{\omega}$-isotropy conditions given in equations $(13,14)$ share a common factor. Setting the common factor to zero, we get

$$
2+2 r_{t}^{2}-z^{2}-4 r_{t} \cos \left(\gamma_{b}-\gamma_{t}\right)=0
$$

Denoting the solutions for $z$ as $z_{\boldsymbol{\omega}}$, the conditions for $\boldsymbol{\omega}$-isotropy can be summarised as

$$
\begin{aligned}
& z_{\boldsymbol{\omega}}= \pm \sqrt{2\left(\left(r_{t}-\cos \left(\gamma_{b}-\gamma_{t}\right)\right)^{2}+\sin ^{2}\left(\gamma_{b}-\gamma_{t}\right)\right)} \\
& \phi=\gamma_{b}-\gamma_{t}
\end{aligned}
$$

The corresponding (positive) leg-lengths are obtained as

$$
L=\sqrt{3\left(\left(r_{t}-\cos \left(\gamma_{b}-\gamma_{t}\right)\right)^{2}+\sin ^{2}\left(\gamma_{b}-\gamma_{t}\right)\right)}
$$

The three equal roots of the characteristic cubic, denoting $\|\boldsymbol{\omega}\|^{2}$, are obtained from equation (10) as

$$
\|\boldsymbol{\omega}\|^{2}=\lambda=\frac{\left(r_{t}-\cos \left(\gamma_{b}-\gamma_{t}\right)\right)^{2}+\sin ^{2}\left(\gamma_{b}-\gamma_{t}\right)}{2 r_{t}^{2} \sin ^{2}\left(\gamma_{b}-\gamma_{t}\right)}
$$


$\boldsymbol{v}$-isotropy - Substituting $\phi=\gamma_{b}-\gamma_{t}$ into equations $(19,20)$, it is possible once again to extract a common factor. Setting the common factor to zero, we obtain

$$
2 z^{2}-\sin ^{2}\left(\gamma_{b}-\gamma_{t}\right)=0
$$

and the manipulator configuration is given by

$$
\begin{aligned}
& z_{\boldsymbol{v}}= \pm \frac{\sin \left(\gamma_{b}-\gamma_{t}\right)}{\sqrt{2}} \\
& L=\sqrt{\left(\left(r_{t}-\cos \left(\gamma_{b}-\gamma_{t}\right)\right)^{2}+\frac{3}{2} \sin ^{2}\left(\gamma_{b}-\gamma_{t}\right)\right.}
\end{aligned}
$$

In this case, we have

$$
\|\boldsymbol{v}\|^{2}=\frac{\left(r_{t}-\cos \left(\gamma_{b}-\gamma_{t}\right)\right)^{2}+\frac{3}{2} \sin ^{2}\left(\gamma_{b}-\gamma_{t}\right)}{3 \sin ^{2}\left(\gamma_{b}-\gamma_{t}\right)}
$$

Combined isotropy - The expressions of $z_{\boldsymbol{\omega}}$ and $z_{\boldsymbol{v}}$ indicate that the solutions are guaranteed to be real numbers for all real values of $r_{t}, \gamma_{b}$ and $\gamma_{t}$. The only difference between these two cases is in the $z$-location of the top platform. Therefore the only additional condition for combined isotropy is simply $z_{\boldsymbol{v}}=z_{\boldsymbol{\omega}}$. Substituting the actual expressions of $z_{\boldsymbol{\omega}}, z_{\boldsymbol{v}}$ from equations $(36,39)$ respectively, we get the condition

$$
4\left(r_{t}-\cos \left(\gamma_{b}-\gamma_{t}\right)\right)^{2}+3 \sin ^{2}\left(\gamma_{b}-\gamma_{t}\right)=0
$$

This equation can be satisfied only when $r_{t}=1$ and $\gamma_{b}=\gamma_{t}\left(\gamma_{b}=\pi+\gamma_{t}\right.$ would require $\left.r_{t}=-1\right)$, i.e., the top and bottom platforms are replicas of each other. As noted earlier, this implies architectural singularity. Further, from the above equations, we get $L=0$, i.e., the top and bottom platforms coincide. This configuration is not of any kinematic interest, and it is the only possible solution in this case.

\subsubsection{Case 2: $\phi=\gamma_{b}-\gamma_{t}+\pi$}

The results for this case are not qualitatively different from the results of case 1, and we briefly summarise them below.

\section{$\omega$-isotropy}

$$
\begin{aligned}
& z_{\boldsymbol{\omega}}= \pm \sqrt{2\left(\left(r_{t}+\cos \left(\gamma_{b}-\gamma_{t}\right)\right)^{2}+\sin ^{2}\left(\gamma_{b}-\gamma_{t}\right)\right)} \\
& L=\sqrt{3\left(\left(r_{t}+\cos \left(\gamma_{b}-\gamma_{t}\right)\right)^{2}+\sin ^{2}\left(\gamma_{b}-\gamma_{t}\right)\right)} \\
& \|\boldsymbol{\omega}\|^{2}=\lambda=\frac{\left(r_{t}+\cos \left(\gamma_{b}-\gamma_{t}\right)\right)^{2}+\sin ^{2}\left(\gamma_{b}-g t\right)}{2 r_{t}^{2} \sin ^{2}\left(\gamma_{b}-\gamma_{t}\right)}
\end{aligned}
$$


$v$-isotropy

$$
\begin{aligned}
& z_{\boldsymbol{v}}= \pm \frac{\sin \left(\gamma_{b}-\gamma_{t}\right)}{\sqrt{2}} \\
& L=\sqrt{\left(\left(r_{t}-\cos \left(\gamma_{b}-\gamma_{t}\right)\right)^{2}+\frac{3}{2} \sin ^{2}\left(\gamma_{b}-\gamma_{t}\right)\right.} \\
& \|\boldsymbol{v}\|^{2}=\frac{\left(r_{t}+\cos \left(\gamma_{b}-\gamma_{t}\right)\right)^{2}+\frac{3}{2} \sin ^{2}\left(\gamma_{b}-\gamma_{t}\right)}{3 \sin ^{2}\left(\gamma_{b}-\gamma_{t}\right)}
\end{aligned}
$$

Combined isotropy - In this case the condition $z_{\boldsymbol{v}}=z_{\boldsymbol{\omega}}$ reduces to the equation

$$
4\left(r_{t}+\cos \left(\gamma_{b}-\gamma_{t}\right)\right)^{2}+3 \sin ^{2}\left(\gamma_{b}-\gamma_{t}\right)=0
$$

This equation can be satisfied only when $r_{t}=1$ and $\gamma_{b}=\gamma_{t}+\pi\left(\gamma_{b}=\gamma_{t}\right.$ would require $\left.r_{t}=-1\right)$. The top and bottom platforms coincide, and the leg lengths are zero.

\subsubsection{Summary: results of family 1}

We summarise below the main results of family 1 .

- For any given set of architectural parameters $\left(r_{t}, \gamma_{b}, \gamma_{t}\right)$, there exist only two configurations (only one with positive $z$ ), such that the Stewart platform is $\boldsymbol{\omega}$-isotropic or $\boldsymbol{v}$-isotropic with all leg lengths equal. Therefore family 1 represents a 3-parameter family of configurations for $\boldsymbol{\omega}$-isotropy and $\boldsymbol{v}$-isotropy individually.

- All the results, such as the singularity conditions, isotropy conditions are functions of the parameter $\gamma=\gamma_{b}-\gamma_{t}$, and $\gamma_{b}, \gamma_{t}$ do not appear individually, or in any other combination. Therefore one of the architectural variables $\gamma_{b}, \gamma_{t}$ is totally decoupled from the isotropy point of view, and can be conveniently used optimally for any other index of performance, such as workspace volume (while maintaining isotropy).

- There are no configurations of kinematic interest at which the manipulator shows $\boldsymbol{\omega}$-isotropy and $\boldsymbol{v}$-isotropy simultaneously.

These observations motivate further generalisations of the assumptions for family 1 , and leads to family 2 of isotropic configurations.

\subsection{Isotropic configurations: family 2}

The assumptions for this family of solutions are the same as in family 1, except that in this case, not all legs are of same length. Instead, the odd numbered legs have identical length $L_{1}$, and the even numbered legs have length $L_{2}=\rho L_{1}$ where $\rho>0$ and in general $\rho \neq 1$. Once again, $\gamma_{b}, \gamma_{t}$ appear only in the group $\left(\gamma_{b}-\gamma_{t}\right)$, and we replace this group by the parameter $\gamma$ below. We treat $\gamma_{t}$ and $\gamma$ as independent variables, and express $\gamma_{b}$ as $\gamma_{b}=\gamma+\gamma_{t}$. 


\subsubsection{Singularity conditions and kinematic constraints}

The determinant of $\boldsymbol{H}$ in this case is given by

$$
D_{\boldsymbol{H}}=\frac{54 r_{t}^{3} z^{3} \cos (\gamma-\phi) \sin (\gamma)}{L_{1}^{6} \rho^{3}}
$$

The numerator is the same as in family 1 , hence the singularity criteria remain the same. Further, $\rho \neq 0$ would be assured if no leg lengths are allowed to reduce to zero.

From equation (23), we obtain only two distinct equations defining the leg lengths:

$$
\begin{aligned}
& L_{1}^{2}=1+r_{t}^{2}+z^{2}-2 r_{t} \cos \phi \\
& \rho^{2} L_{1}^{2}=1+r_{t}^{2}+z^{2}-2 r_{t} \cos (2 \gamma-\phi)
\end{aligned}
$$

Eliminating $L_{1}$ between the above equations, we get a linear equation in $\rho^{2}$, which gives the positive solution for $\rho$ as

$$
\rho=\sqrt{\frac{\left(r_{t}-\cos (2 \gamma-\phi)\right)^{2}+z^{2}+\sin ^{2}(2 \gamma-\phi)}{\left(r_{t}-\cos \phi\right)^{2}+\sin ^{2} \phi}+z^{2}}
$$

The corresponding solution of $L_{1}$ is obtained as

$$
L_{1}=\sqrt{\left(r_{t}-\cos (2 \gamma-\phi)\right)^{2}+z^{2}+\sin ^{2}(2 \gamma-\phi)}
$$

The expressions for $\rho, L_{1}$ indicate that there are five free parameters, namely $r_{t}, \gamma, \gamma_{t}, \phi$ and $z$, for which the kinematic constraints are valid. We now search for isotropic configurations within this 5-parameter family of kinematically valid configurations. First, we establish the conditions for isotropy in general.

\subsubsection{Conditions for isotropy}

$\boldsymbol{\omega}$-isotropy - The equations resulting from substitution of the expression of $\rho$ from equation (42) into $\boldsymbol{\omega}$-isotropy conditions $(13,14)$ are found to share a common factor, which can be written as a polynomial in $z_{\boldsymbol{\omega}}\left(z_{\boldsymbol{\omega}}\right.$ denotes the vertical displacement of $\boldsymbol{p}$ in this case):

$$
z_{\boldsymbol{\omega}}^{4}+c_{1} z_{\boldsymbol{\omega}}^{2}+c_{2}=0
$$

where

$$
\begin{aligned}
& c_{1}=-\cos (2(\gamma-\phi))\left(r_{t}^{2}-2 \cos (\gamma) \cos (\gamma-\phi) r_{t}+1\right) \\
& c_{2}=-2 \cos ^{2}(\gamma-\phi)\left(r_{t}^{2}-2 \cos (2 \gamma-\phi) r_{t}+1\right)\left(r_{t}^{2}-2 \cos (\phi) r_{t}+1\right)
\end{aligned}
$$

It may be seen easily that $c_{2} \leq 0$, and hence the discriminant of the above quadratic in $z_{\boldsymbol{\omega}}^{2}$ is $c_{1}^{2}-4 c_{2} \geq 0$. It can also be seen that one of the solutions for $z_{\omega}^{2}$ is always positive, and the other negative. Therefore, we conclude that for any given set of architectural parameters $r_{t}, \gamma_{t}, \gamma$ and rotation $\phi$, there exist a unique positive value of $z_{\boldsymbol{\omega}}$ and a negative value of equal magnitude such that $\boldsymbol{\omega}$-isotropy conditions are met. These paired solutions correspond to the mirror reflections at the base plane. 
$\boldsymbol{v}$-isotropy - In this case also, substituting for $\rho$ into the $\boldsymbol{v}$-isotropy conditions $(19,20)$, we obtain a common zero factor as a quadratic in $z_{\boldsymbol{v}}^{2}\left(z_{\boldsymbol{v}}\right.$ denotes the vertical displacement of $\boldsymbol{p}$ in this case):

$$
d_{0} z_{\boldsymbol{v}}^{4}+d_{1} z_{\boldsymbol{v}}^{2}+d_{2}=0
$$

where

$$
\begin{aligned}
d_{0}= & 4(\cos (2 \gamma-2 \phi)+1) \\
d_{1}= & 4(\cos (2 \gamma-2 \phi)+1) r_{t}^{2}-2(\cos (2 \gamma-3 \phi)+\cos (4 \gamma-3 \phi)+3 \cos (2 \gamma-\phi)+3 \cos (\phi)) r_{t}+ \\
& 4 \cos (2 \gamma-2 \phi)+\cos (4 \gamma-2 \phi)+\cos (2 \phi)-2 \\
d_{2}= & (\cos (4 \gamma-2 \phi)+\cos (2 \phi)-2) r_{t}^{2}-(\cos (2 \gamma-3 \phi)+\cos (4 \gamma-3 \phi)-2 \cos (2 \gamma-\phi)- \\
& \cos (4 \gamma-\phi)-2 \cos (\phi)+\cos (2 \gamma+\phi)) r_{t}+\cos (4 \gamma-2 \phi)+\cos (2 \phi)-2
\end{aligned}
$$

Real solutions of $z_{\boldsymbol{v}}^{2}$, would exist if $d_{1}^{2}-4 d_{0} d_{2} \geq 0$. In this case, clearly $d_{0} \geq 0$, therefore it suffices to prove $d_{2} \leq 0$. We find that $d_{2}$ is of the form $q_{0} r_{t}^{2}+q_{1} r_{t}+q_{2}$, where $q_{0}=q_{2}=(\cos (4 \gamma-2 \phi)+$ $\cos (2 \phi)-2) \leq 0$. It can be shown that $d_{2}$ has the same sign as $q_{0}$ if $q_{1}^{2}-4 q_{0} q_{2} \leq 0$. Indeed, $q_{1}^{2}-4 q_{0} q_{2}=8(2 \cos (2 \gamma)+\cos (2 \phi)+\cos (4 \gamma-2 \phi)+2 \cos (2(\gamma-\phi))-6) \sin ^{2}(2 \gamma-\phi) \sin ^{2}(\phi) \leq 0$. Therefore $d_{2} \leq 0$, and hence equation (46) has a pair of real solutions in $z_{v}^{2}$. It can be further shown that under these conditions, one of the roots is always positive, and the other negative, therefore we have a negative and a positive solution of same magnitude in $z_{\boldsymbol{v}}$. This shows that there is a 4-parameter family of solutions for $\boldsymbol{v}$-isotropy in the parameters $\gamma_{t}, \gamma, r_{t}$ and $\phi$, and for each combination of the parameters, $\boldsymbol{v}$-isotropy occurs at a unique positive value of $z$.

Combined isotropy - The additional condition for combined isotropy is the same as in family 1: $z_{\boldsymbol{v}}=z_{\boldsymbol{\omega}}$. In other words, equations $(44,46)$ should have common root(s). The condition for the same can be obtained in closed-form by application of elementary elimination theory [31]:

$$
c_{2}^{2} d_{0}^{2}+c_{1} c_{2} d_{0} d_{1}+c_{1}^{2} d_{0} d_{2}-c_{1} d_{1} d_{2}+d_{2}^{2}+c_{2}\left(d_{1}^{2}-2 d_{0} d_{2}\right)=0
$$

The common root is obtained as

$$
z_{\boldsymbol{\omega}}^{2}=z_{\boldsymbol{v}}^{2}=\frac{d_{2}-d_{0} c_{2}}{d_{1}-d_{0} c_{1}}
$$

Note that the expression for the common root above is valid iff equation (48) holds. Upon substitution of the actual expressions of $c_{i}, d_{i}$, equation (48) results in a 6 th degree polynomial in $r_{t}$. However, it is possible to factor-out the singular term $r_{t} \sin ^{2}(\gamma)$ using the monomial-based canonical form of the expression [19]. The reduced equation is a parametric quintic of the following form:

$$
f_{0} r_{a}^{5}+f_{1} r_{a}^{4}+f_{2} r_{a}^{3}+f_{3} r_{t}^{2}+f_{4} r_{t}+f_{5}=0
$$

where each of the coefficients $f_{i}$ are functions of the two parameters ${ }^{6}: \gamma$, and $\phi$. Recall that the complex roots of an equation with real coefficients are pairwise conjugate, hence it is ensured that equation (50) would have 1, 3 or 5 real root(s) for any real combination of $\gamma$, and $\phi$. The number of real roots can be estimated from the coefficients, using Descartes' rule of signs or from the

\footnotetext{
${ }^{6}$ The expressions of $f_{i}$ have been obtained symbolically, but are not displayed here due to their large size.
} 
Sturm sequence. However, due to the complicated nature of the coefficients, this can only be done numerically. It is also possible to ascertain the numerical bounds on the parameters $\gamma, \phi$ such that $r_{t}$ lies within given bounds using interval analysis [32,33]. The roots themselves can only be obtained numerically, since it is not possible to solve a general algebraic equation of degree more than 4 in closed-form [27].

The steps to compute the combined isotropy configurations are the following:

1. Solve equation (50) for a given numeric pair of values $(\gamma, \phi)$.

2. Extract the positive root(s) among the 5 solutions obtained above.

3. For each of the feasible value(s) of $r_{t}$, compute the value(s) of $z$ from equation (49), and extract only the positive one(s) from them.

4. Using the valid set(s) of $\left(r_{t}, z\right)$, compute $\rho, L_{1}$ from equation $(42,43)$ respectively.

In this way we obtain all the unknowns in the manipulator architecture and configuration. We demonstrate the procedure with numerical examples below.

\subsubsection{Examples of combined isotropy}

We choose the free parameters as

$$
\gamma_{b}=\frac{2 \pi}{15}, \gamma_{t}=\frac{\pi}{18}, \phi=\frac{2 \pi}{5}
$$

The solutions for $r_{t}$ are obtained from equation (50) as

$$
r_{t}=(0.5575,0.7482,0.845267-0.758591 i, 0.845267+0.758591 i, 0.8939)
$$

The corresponding values of $z$ from equation (49) are

$$
z=(0.6863,0.6858 i, 0.6955+0.3838 i, 0.6955-0.3838 i, 0.6284)
$$

It can be seen that only the first and the last values in each case are feasible. The corresponding configurations are shown below in figures $2(\mathrm{a}), 2(\mathrm{~b})$.

\subsubsection{Spatial isotropy}

In this section, we investigate whether family 2 admits any spatially isotropic configuration as per the definition of Klein and Miklos [9]. This requires combined isotropy, and the additional condition $\boldsymbol{J}_{\boldsymbol{\omega}} \boldsymbol{J}_{\boldsymbol{v}}^{T}=\mathbf{0}$. For family 2 , we find that

$$
\boldsymbol{J}_{\boldsymbol{\omega}} \boldsymbol{J}_{\boldsymbol{v}}^{T}=\left(\begin{array}{ccc}
k_{11} & k_{12} & 0 \\
-k_{12} & k_{22} & 0 \\
0 & 0 & k_{33}
\end{array}\right)
$$




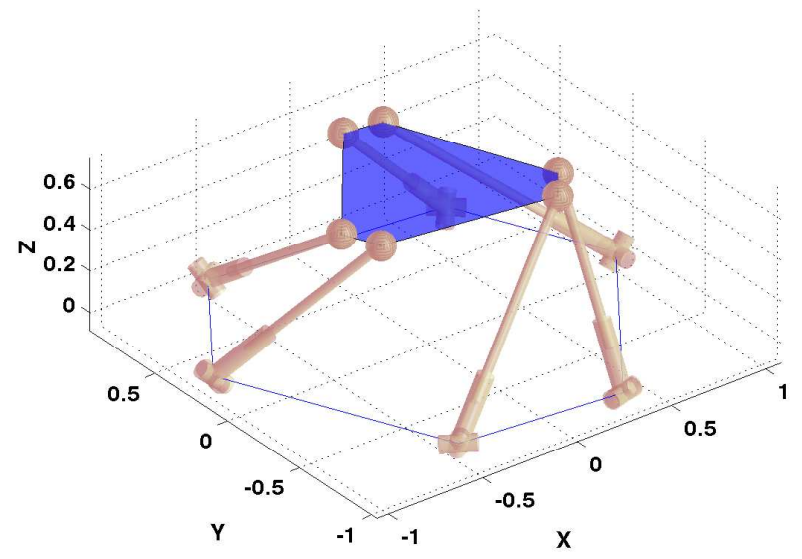

(a) $r_{t}=0.5575, z=0.6863$

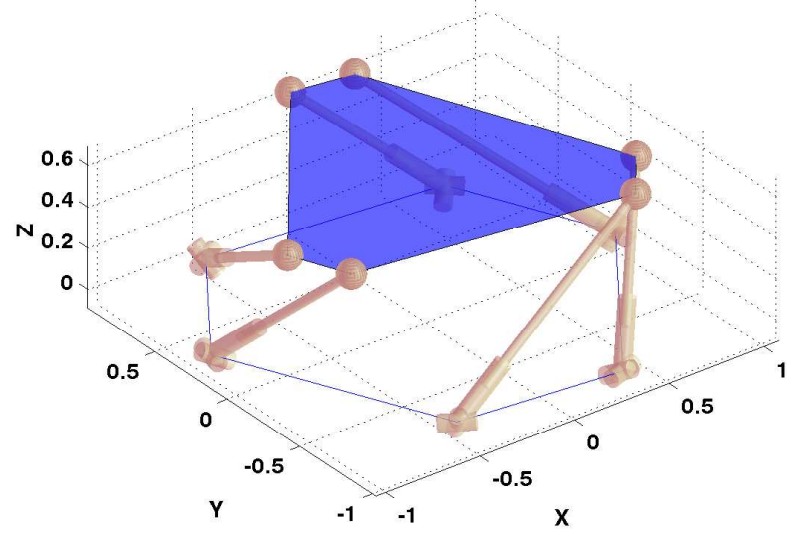

(b) $r_{t}=0.8939, z=0.6284$

Figure 2: Combined isotropy configurations of the SRSPM

where

$$
\begin{aligned}
& k_{11}=-\frac{\csc ^{2}(\gamma)\left(\sin (2 \gamma-\phi)-\rho^{2} \sin (\phi)\right) L_{1}^{2}}{6 z r_{t}} \\
& k_{12}=\frac{\csc ^{2}(\gamma) L_{1}^{2}\left(\cos (\phi) \rho^{2}+\cos (2 \gamma-\phi)-\left(\rho^{2}+1\right) r_{t}\right)}{6 z r_{t}} \\
& k_{22}=-\frac{\csc ^{2}(\gamma)\left(\sin (2 \gamma-\phi)-\rho^{2} \sin (\phi)\right) L_{1}^{2}}{6 z r_{t}} \\
& k_{33}=\frac{\csc ^{2}(\gamma) \sec ^{2}(\gamma-\phi)\left(\sin (2 \gamma-\phi)-\rho^{2} \sin (\phi)\right) L_{1}^{2}}{12 z r_{t}}
\end{aligned}
$$

It can be seen that the diagonal entries, $k_{i i}$, share a common factor, $\left(\sin (2 \gamma-\phi)-\rho^{2} \sin (\phi)\right)$. Setting it to zero, we get,

$$
\rho= \pm \sqrt{\csc (\phi) \sin (2 \gamma-\phi)}
$$

Using the positive value of $\rho$, we solve for $r_{t}$ from the equation $k_{12}=0$ :

$$
r_{t}=\cos (\gamma) \sec (\gamma-\phi)
$$

We already have the expression of $\rho$ from the kinematic constraints for family 2 of configurations (see equation (42)). Setting it equal to the expression in equation (52), and substituting $r_{t}$ from equation (53), we can find $z$ as

$$
z= \pm \frac{\sqrt{\sin (2 \gamma-\phi) \sin (\phi)}}{\cos (\gamma-\phi)}
$$

Using the expressions of $\rho, r_{t}$ and $z$ (positive value) from equations $(52,53,54)$ respectively in the $\boldsymbol{\omega}$-isotropy equations $(13,14)$, we find that both of these are satisfied when we have

$$
\phi=\gamma \pm \frac{\pi}{4}
$$


The conditions for $\boldsymbol{v}$-isotropy (equations $(19,20)$ ) are found to be satisfied when we have

$$
1+\cot (\gamma) \cot (\phi)=0
$$

The above condition implies that $\phi=\gamma \pm \frac{\pi}{2}$, which defines a singularity as noted earlier.

It is therefore conclusive that no non-singular configuration showing spatial isotropy is achievable within family 2 configurations of the SRSPM.

\subsubsection{Summary: results of family 2}

The main results of family 2 of isotropic configurations are the following:

- An SRSPM of any architecture defined by the set $r_{t}, \gamma_{b}, \gamma_{t}$ has a 1-parameter family of solutions in the parameter $\phi$ for the $\boldsymbol{\omega}$-isotropy, and $\boldsymbol{v}$-isotropy respectively. The possible configuration is unique for each set of the 4 parameters in either case, and the $z$ locations differ in general in the two cases.

- It is possible to attain $\boldsymbol{\omega}$-isotropy and $\boldsymbol{v}$-isotropy simultaneously in the class of Stewart platforms considered. Family 2 represents a 3-parameter family of solutions in the parameters $\gamma, \phi$ and $\gamma_{t}$. However, while $\gamma_{t}$ is a free parameter in this context, not all combinations of the other two lead to real or feasible solutions.

- There exists no spatially isotropic SRSPM within the family 2 of configurations which is non-singular.

\section{Design of an SRSPM for combined isotropy}

In the previous section, we have studied isotropic configurations in an SRSPM. In this section, we discuss two important design problems: (a) synthesis of an SRSPM for combined isotropy at a given location and orientation, and (b) obtaining the combined isotropy points of an SRSPM of a given architecture. We show that both the problems can be solved within the family 2 of configurations described in the previous section.

\subsection{Synthesis of an SRSPM for combined isotropy at a given position $z_{0}$ and orientation $\phi_{0}$}

In this case we assume that the top platform location and orientation have been completely specified by $z_{\boldsymbol{\omega}}=z_{\boldsymbol{v}}=z_{0}$ and $\phi=\phi_{0}$ in conjunction with the assumptions defining the family 2 . The task is to obtain $\gamma$ and $r_{t}$ such that the manipulator is isotropic in the combined sense.

We start with the $\boldsymbol{\omega}$-isotropy equation (44) and the $\boldsymbol{v}$-isotropy equation (46). Substituting the actual expressions of $c_{i}, d_{i}$ from equations $(45,47)$ in these equations, and rewriting them as polynomial equations in $r_{t}$, we get a quartic and a quadratic respectively:

$$
\begin{aligned}
& g_{0} r_{t}^{4}+g_{1} r_{t}^{3}+g_{2} r_{t}^{2}+g_{3} r_{t}+g_{4}=0 \\
& h_{0} r_{t}^{2}+h_{1} r_{t}+h_{2}=0
\end{aligned}
$$


The common root of these two equations can be explicitly written in terms of the coefficients $g_{i}, h_{i}$ as

$$
r_{t}=\frac{h_{2}\left(g_{2} h_{0}^{2}-g_{1} h_{0} h_{1}+g_{0}\left(h_{1}^{2}-h_{0} h_{2}\right)\right)-h_{0}^{3} g_{4}}{g_{3} h_{0}^{3}-g_{2} h_{0}^{2} h_{1}+h_{1}^{2}\left(g_{1} h_{0}-g_{0} h_{1}\right)-h_{0} h_{2}\left(g_{1} h_{0}-2 g_{0} h_{1}\right)}
$$

Equation (58) gives the correct expression for the common root in $r_{t}$ when the resultant of the two equations in (57) with respect to $r_{t}$ vanishes [31]. The resultant is a complicated expression involving trigonometric terms in $\gamma$, and algebraic terms in $z_{0}$. We convert it into a polynomial with explicit coefficients using symbolic computation. The result is a 44 th degree polynomial in $t$. Using the monomial-based canonical form again, we factor out the term $t^{4}$ from the polynomial, thereby reducing its degree to 40.

Extracting the real values of $t$ such that the corresponding values of $\gamma$ are within the prescribed limits, we compute $r_{t}$ from equation (58). For every $r_{t}>0$ within the specified range, the free parameter $\gamma_{t}$ can be chosen as convenient, and the architecture of the manipulator can be completely prescribed. We illustrate this synthesis procedure with an example below.

\subsubsection{Example of combined isotropy at a given configuration}

We choose the configuration as $z_{0}=1 / 2, \phi_{0}=\pi / 2$, and the free architectural parameter as $\gamma_{t}=\pi / 18$. Corresponding to these values, there are 18 real solutions for $t$, of which, however, only one turns out to be feasible. The corresponding values of $\gamma$ and $r_{t}$ are 0.4997 and 0.7790 respectively. The configuration is shown in figure 3 .

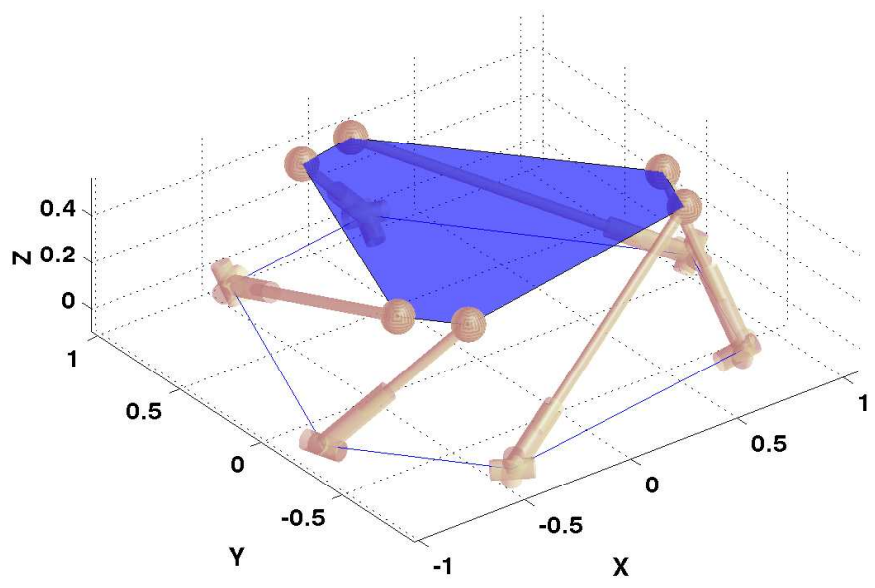

Figure 3: Isotropic configuration of the SRSPM at a given configuration

\subsection{Isotropic configurations of an SRSPM of given architecture}

In this section, we solve the problem complementary to the above synthesis procedure, i.e., we find out the isotropic configurations of an SRSPM of given geometry. In this case, the manipulator geometry is completely specified in terms of the architectural variables, $r_{t}, \gamma$ and $\gamma_{t}$. We need to find the configuration variables $z$ and $\phi$ such that the conditions for combined isotropy are met. 
This type of analysis is very useful for determining the optimal working configurations of existing manipulators.

We refer to the condition for combined isotropy in equation (48), which is a function of $\phi$ alone. We convert this equation into a polynomial in $u=\tan (\phi / 2)$ following the steps mentioned in the previous section. In this case we end up with a 22 -degree polynomial in $u$. For each of the feasible values of $\phi$ arising from the solutions for $u$, the corresponding value of $z$ can be computed uniquely from equation (49), thereby completing the definition of the manipulator configuration. We demonstrate the solution procedure with two examples below.

\subsubsection{Examples of combined isotropy in existing SRSPMs}

Example 1: Based on the INRIA prototype. In this example, the geometry of the top and bottom platforms are taken to be the same as the INRIA prototype (data adopted from [34]). However, the top platform is mobile in our case, and all the lengths are scaled such that the radius of the bottom platform is unity. The resulting architectural parameters of this manipulator are as follows:

$$
r_{t}=0.5803, \quad \gamma_{b}=0.2985, \quad \gamma_{t}=0.6573
$$

and therefore $\gamma=-0.3588$. The manipulator is shown in figure 4(a) below in a reference configuration, where $x=y=0, z=1$, and $\boldsymbol{R}=\boldsymbol{R}_{z}(\gamma)$, such that the corresponding axes of symmetry in the two platforms are vertically aligned. The isotropic configurations are shown in figures 4(b)-4(e).

Example 2: Based on the University of Harbin prototype We obtain the combined isotropy configurations of the University of Harbin prototype in this case (see [24] for details). The reduced architectural parameters are

$$
r_{t}=0.8939, \gamma_{b}=0.4189, \gamma_{t}=0.1745, \gamma=0.2443
$$

We show the reference configuration of the manifold in figure 5(a), and the set of isotropic configurations in figures $5(\mathrm{~b})-5(\mathrm{e})$.

\section{Sensitivity of the isotropic configurations to variations in the configuration parameters of the moving platform}

The isotropic configurations discussed and obtained in this paper are local, i.e., at a configuration. From the point of view of actual application of an SRSPM, the local isotropic configurations are of limited use-ideally, we would like to have isotropy or near-isotropy condition over an extended region in the workspace. In other words, robustness of the isotropy condition to small changes in architectural parameters is a desired property. In this section, we present a few representative numerical results describing the departure from isotropy behavior with variations in the configuration parameters defining isotropy. The configuration space of an SRSPM is the 6-dimensional manifold $S E(3)$, and hence we can only visualise the variations in different sections of $S E(3)$. In the following discussion, we present 3 different combinations of configuration variations:

- Variation in $x, y$ : The top platform remains in a single horizontal plane, and retains its orientation. 


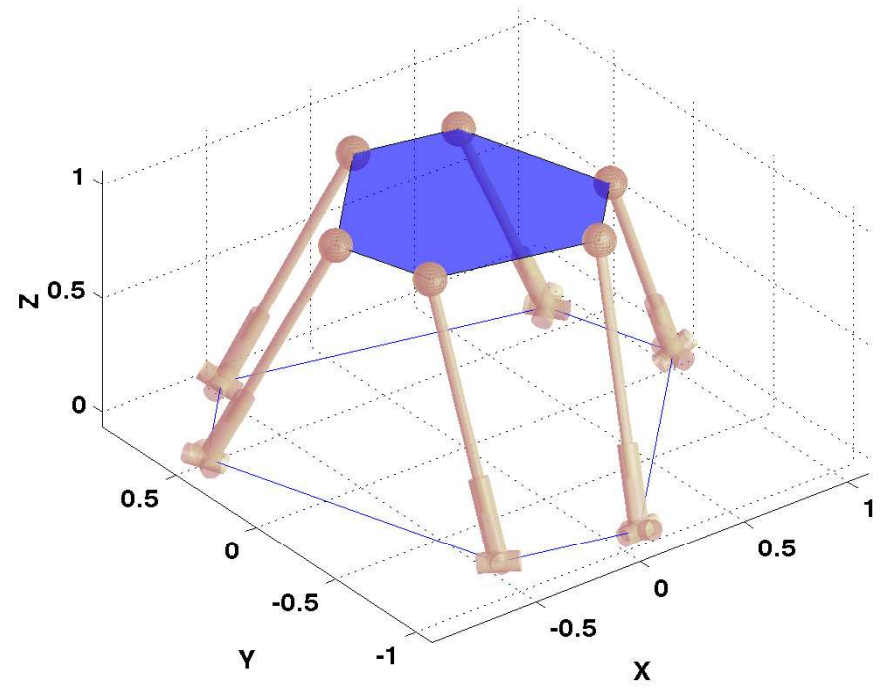

(a) Reference configuration $(z=1, \phi=\gamma)$

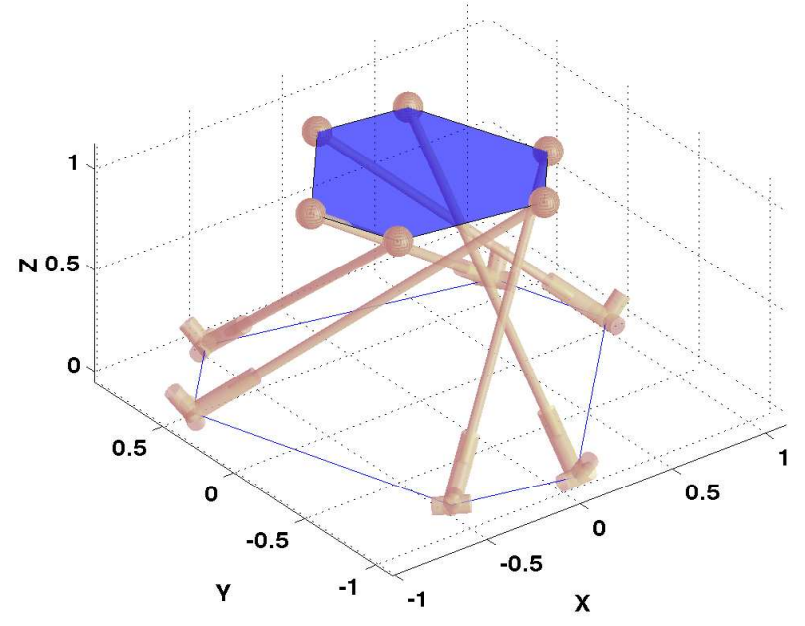

(b) $z=1.0669, \phi=1.7921$

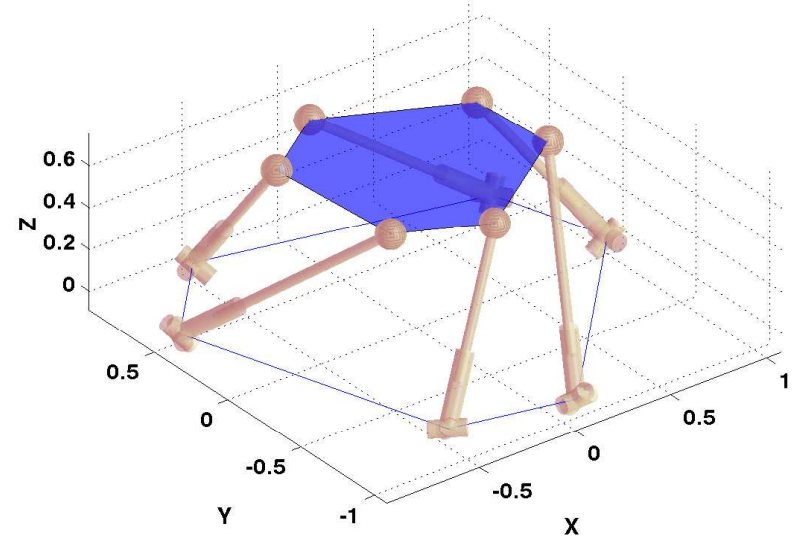

(d) $z=0.6894, \phi=0.4657$

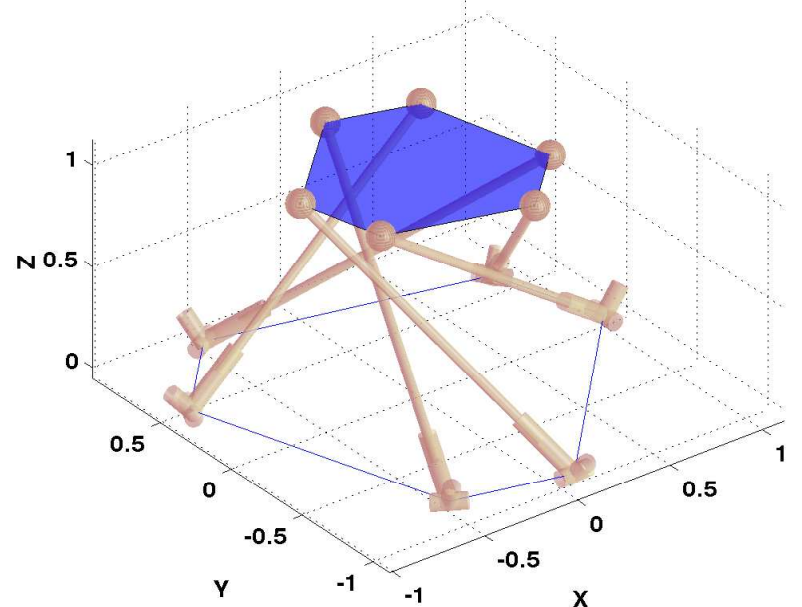

(c) $z=1.0669, \phi=-2.5097$

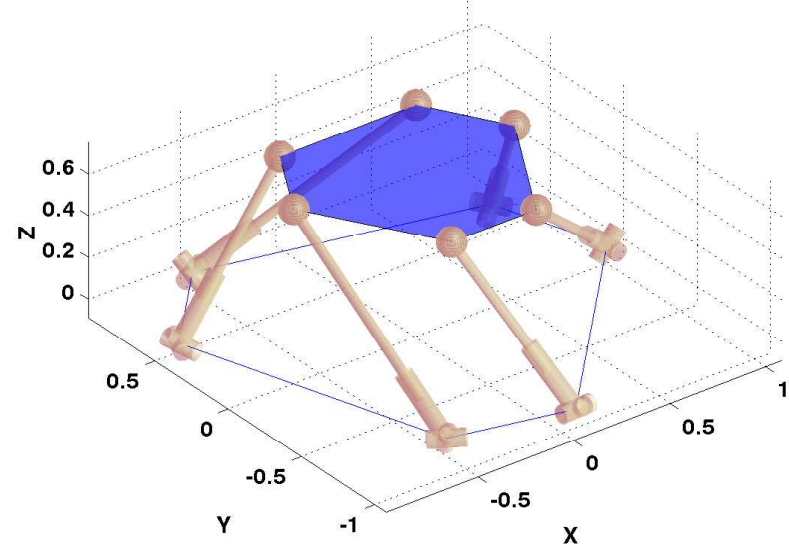

(e) $z=0.6894, \phi=-1.1833$ 


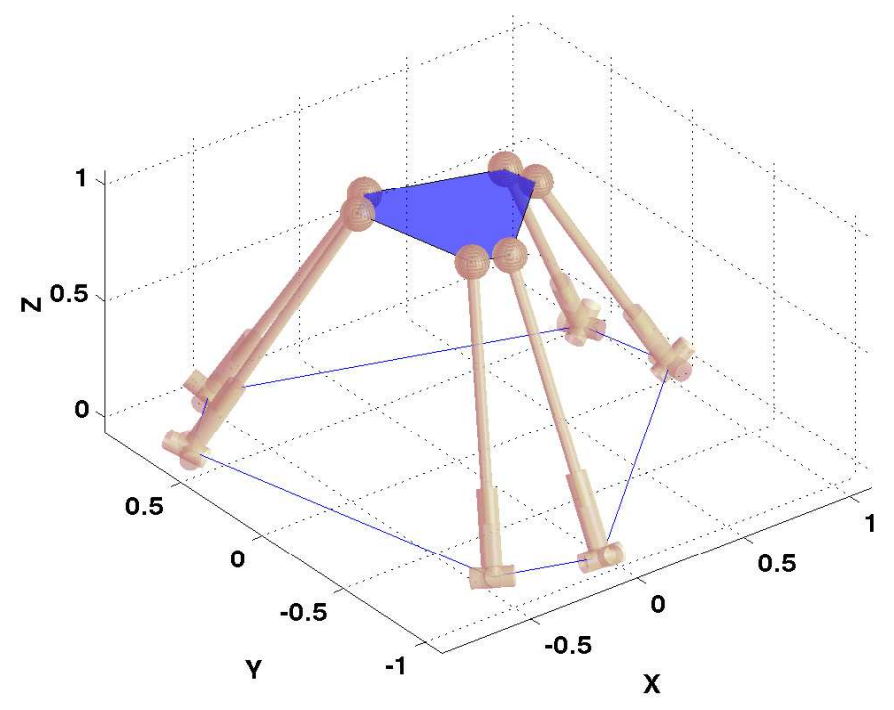

(a) Reference configuration, $z=1, \phi=\gamma$

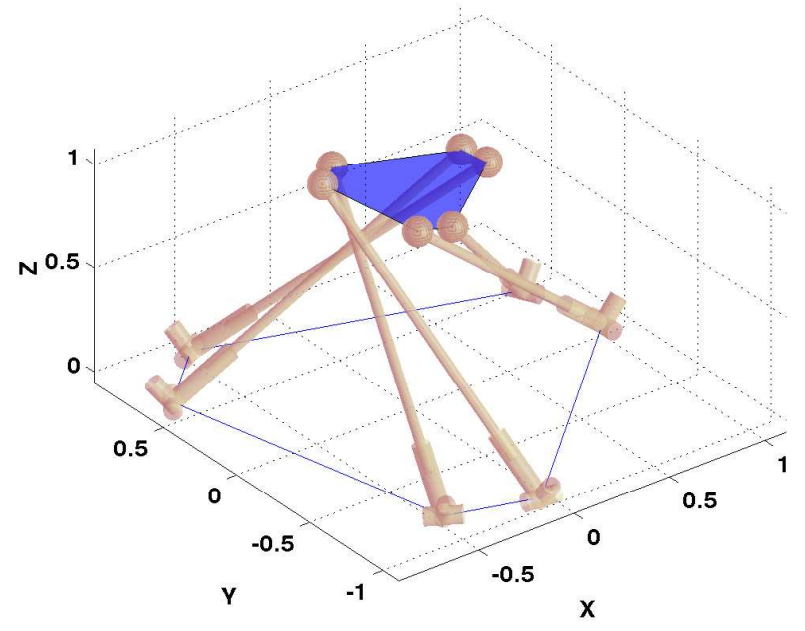

(b) $z=1.0130, \phi=-2.1643$

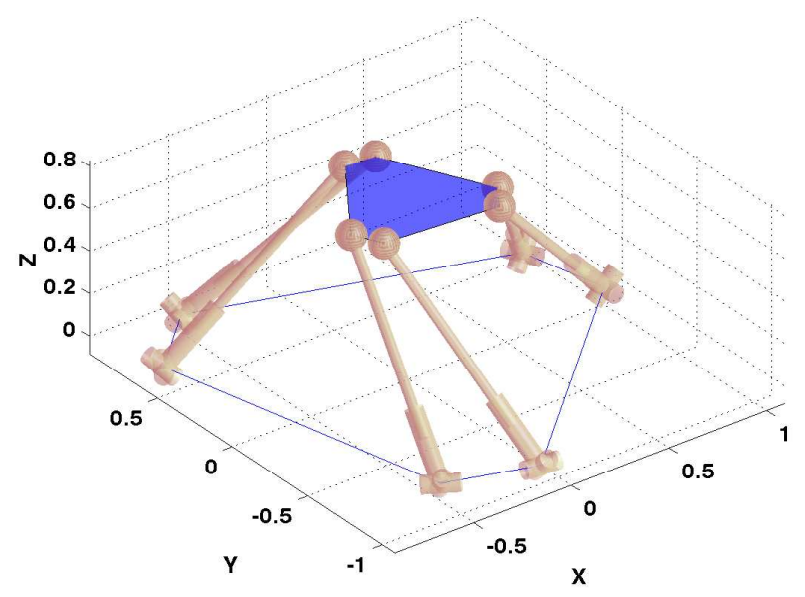

23

(d) $z=0.7639, \phi=-0.8082$

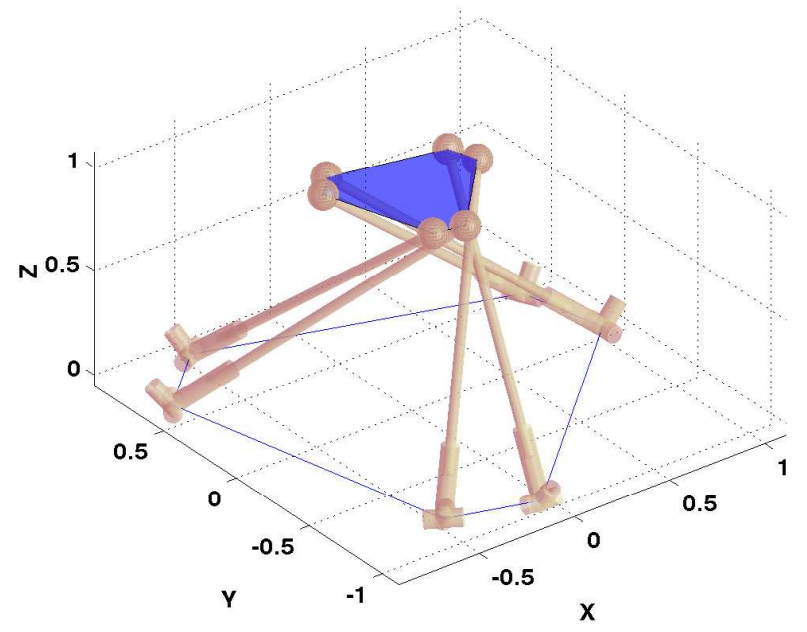

(c) $z=1.0130, \phi=2.1961$

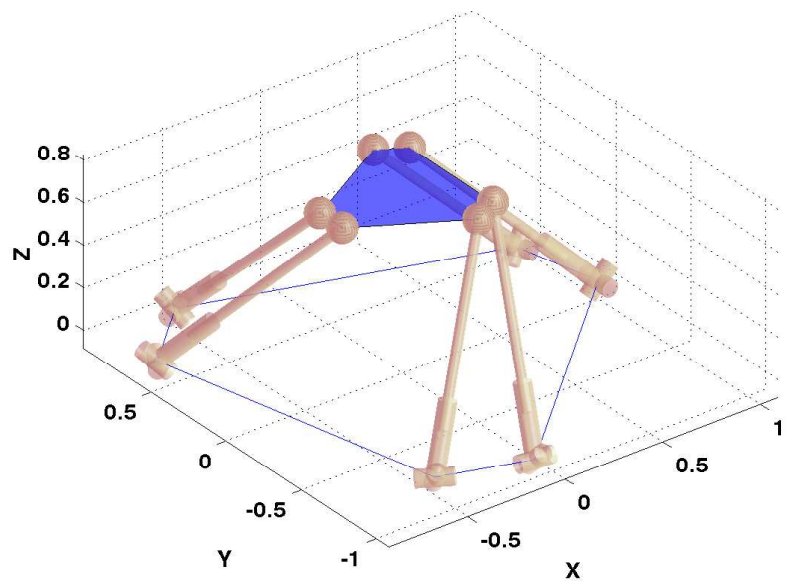

(e) $z=0.7639, \phi=0.8399$ 
- Variation in $z, \phi$ : The top platform remains horizontal and is located directly above the origin. It is allowed to rotate only about the $Z$ axis.

- Variation in $\theta_{x}, \theta_{y}$ : The location of the top platform remains unchanged, while it is rotated about the orientation at its home position. In this case the orientation of the moving platform is given by $\boldsymbol{R}_{z}(\phi) \boldsymbol{R}_{x}\left(\theta_{x}\right) \boldsymbol{R}_{y}\left(\theta_{y}\right)$ in the fixed reference frame.

In section 5.2, we have seen that the isotropic configurations occur in pairs in a plane, i.e., there are two solutions for the rotation angle $\phi$ such that isotropy conditions are met (see figures $(4(\mathrm{~b}), 4(\mathrm{c}))$ and figures $(4(\mathrm{~d}), 4(\mathrm{e})))$. We first take a look at the sensitivity to variation in position around these two configurations, then proceed to study the other variations in the better of the two. In all of the following analysis, we use the INRIA design for the top and bottom platforms (as in section 5.2). The measures of deviation from angular and linear isotropy are quantified in terms of the departure of the condition numbers $\kappa_{2}\left(\boldsymbol{J}_{\boldsymbol{\omega}}\right), \kappa_{2}\left(\boldsymbol{J}_{\boldsymbol{v}}\right)$ respectively from unity.

\subsection{Sensitivity to $x, y$}

We vary both $x, y$ from $-1 / 2$ to $1 / 2$ about the isotropic configuration at $x=y=0^{7}$. We plot the quantity $\delta_{\boldsymbol{\omega}} \triangleq \kappa_{2}\left(\boldsymbol{J}_{\boldsymbol{\omega}}\right)-1$ over the above-mentioned range of $x, y$ in figures $6(\mathrm{a})$, and $6(\mathrm{~b})$ for the isotropic configurations at $z=1.0669, \phi=-2.5097$ (figure $4(\mathrm{c})$ ), and $z=0.6894, \phi=$ 0.4657 (figure $4(\mathrm{e})$ ) respectively. In figures $6(\mathrm{c})$, and $6(\mathrm{~d})$, we plot $\delta_{\boldsymbol{v}} \triangleq \kappa_{2}\left(\boldsymbol{J}_{\boldsymbol{v}}\right)-1$ for the corresponding cases. The other halves of the paired configurations (see figures $(4(\mathrm{~b}), 4(\mathrm{~d}))$ ) at the same value of $z$ are not discussed here, as they represent nearly same qualitative and quantitative behavior. The following observations can be made from these plots:

- The isotropic configuration at $x=y=0$ seems to be very stable, as the condition numbers appear to show convexity about this point.

- The isotropic configuration is the global minima of the deviation within the given range in each case.

- The sensitivity to $x, y$ is much lower for the case with $z=1.0669$ than in the other for both the angular and linear cases, with condition numbers varying up to 2.1 and 3.3 respectively in this case, as compared to about 14 and 11 respectively in the other case.

We study the better configuration from the above for the rest of this section. First, we vary the $z$ location to 0.6 times to 1.4 times of its isotropy value $z_{0}=1.0669$ in steps of 0.2 , and present the sensitivity plots with respect to $x, y$. Figures $7(\mathrm{a})-7(\mathrm{e})$ show the contours of $\delta_{\boldsymbol{\omega}}$, with the ' + ' sign marking the point $x=y=0$. It may be concluded from these figures that for $z \in\left[0.6 z_{0}, 1.2 z_{0}\right]$, $\boldsymbol{\omega}$-isotropy is relatively insensitive to variations in $x, y$. Figures $8(\mathrm{a})-8(\mathrm{e})$ show similar trends in the corresponding contours of $\delta_{\boldsymbol{v}}$.

\footnotetext{
${ }^{7}$ As noted earlier in section 3 , all the linear dimensions are scaled by the base radius. Therefore $x=1 / 2$ in this case implies that the top platform center has moved out by half the base radius in the $X$ direction, which is quite representative as a range for such manipulators.
} 


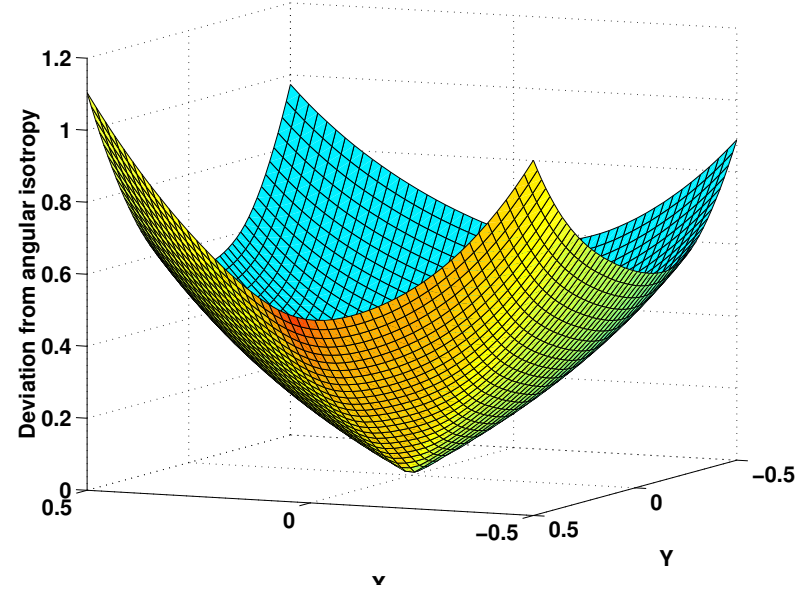

(a) Departure from angular isotropy $(z=$ $1.0669, \phi=-2.5097)$

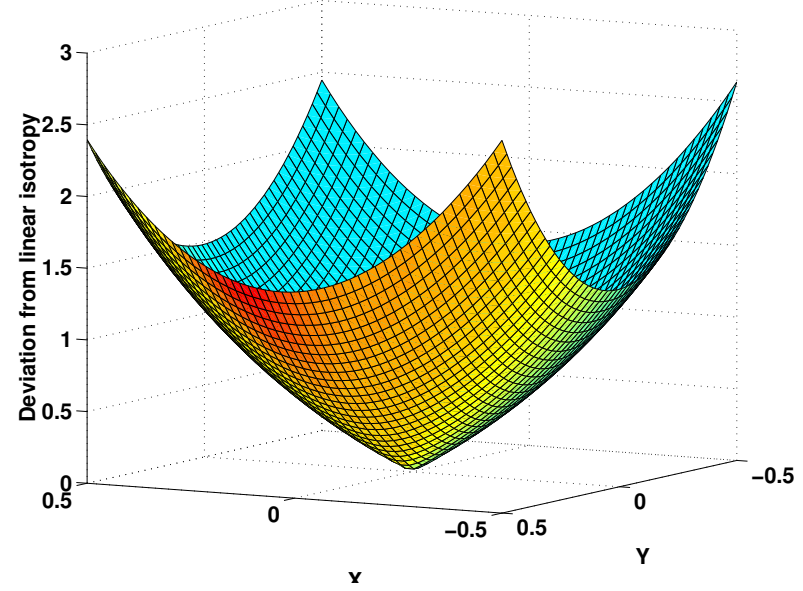

(c) Departure from linear isotropy $(z=$ $1.0669, \phi=-2.5097)$

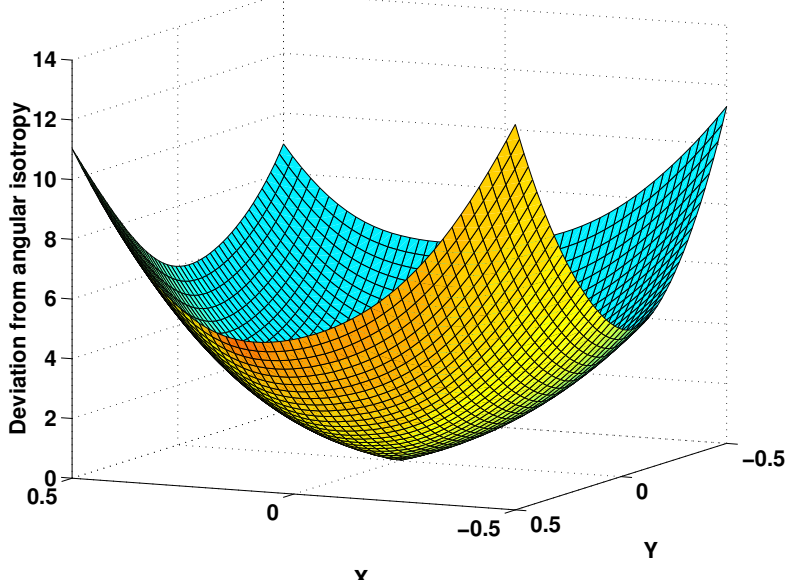

(b) Departure from angular isotropy $(z=$ $0.6894, \phi=-1.1833)$

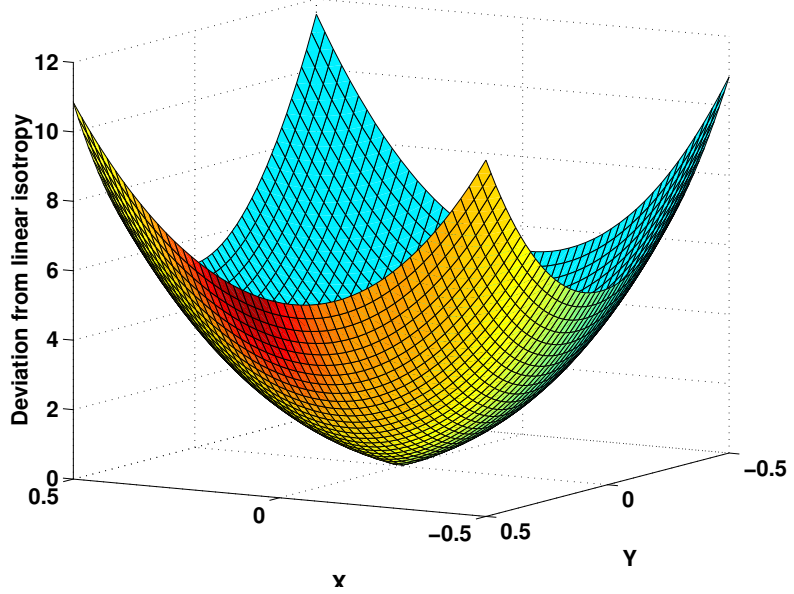

(d) Departure from linear isotropy $(z=$ $0.6894, \phi=-1.1833)$

Figure 6: Sensitivity to $x, y$ in two combined isotropy configurations of the SRSPM with INRIA geometry 


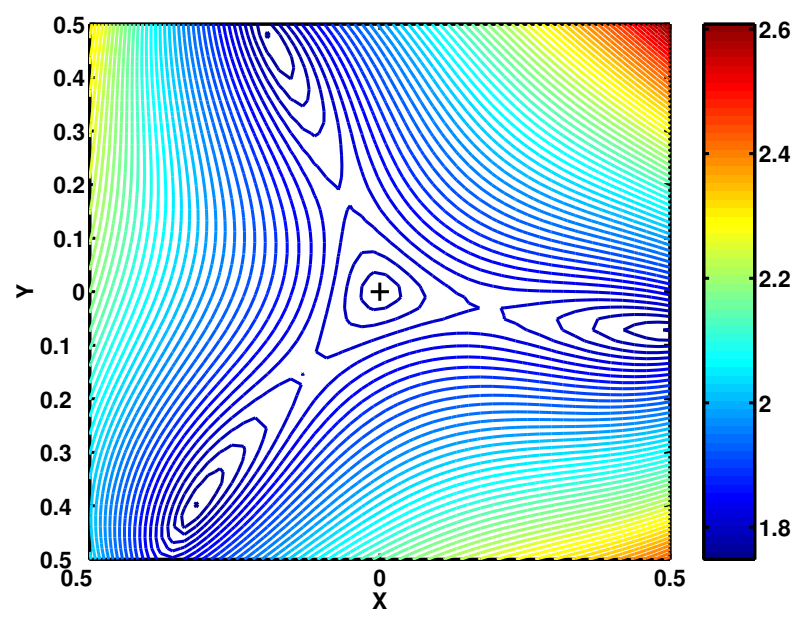

(a) $z=0.6 z_{0}$

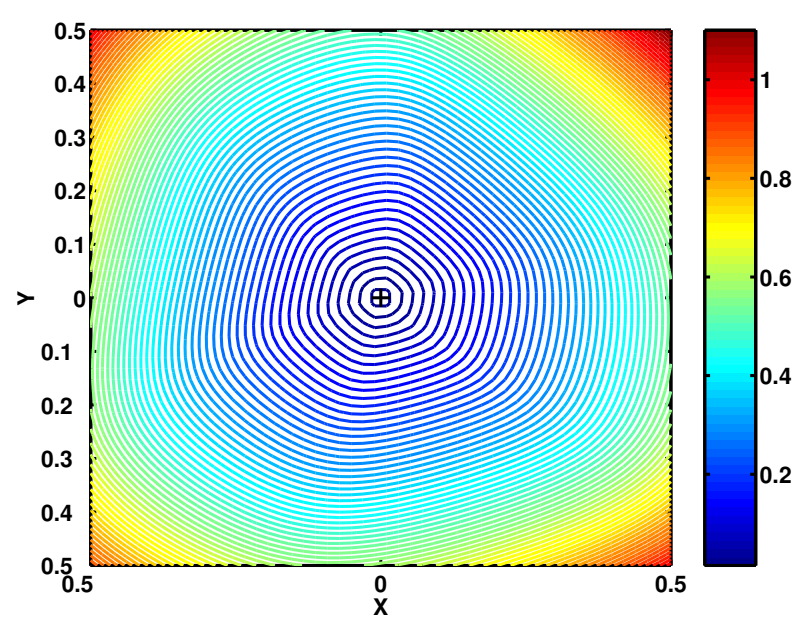

(c) $z=z_{0}$

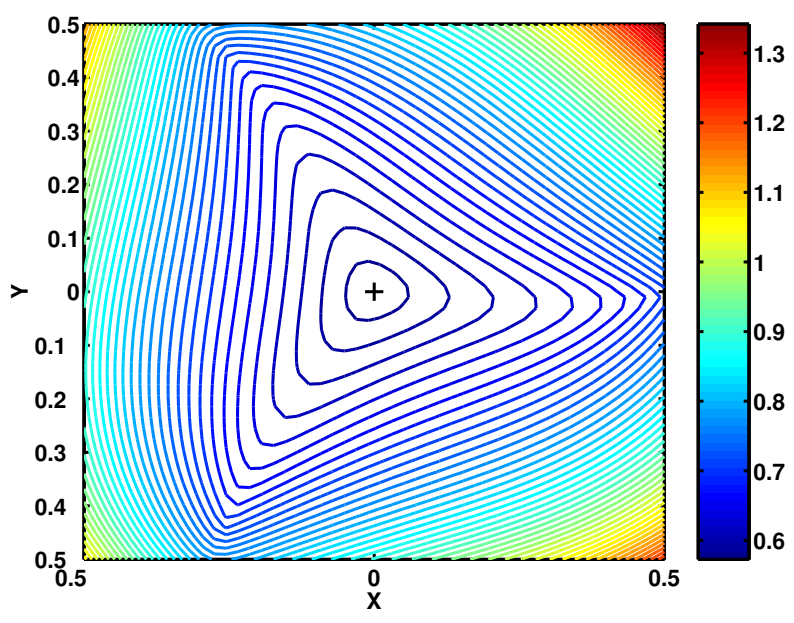

(b) $z=0.8 z_{0}$

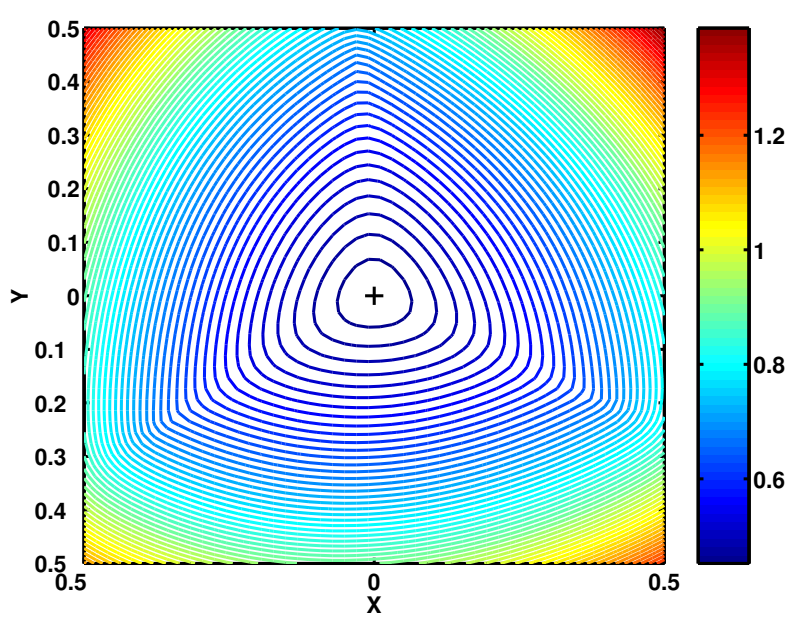

(d) $z=1.2 z_{0}$

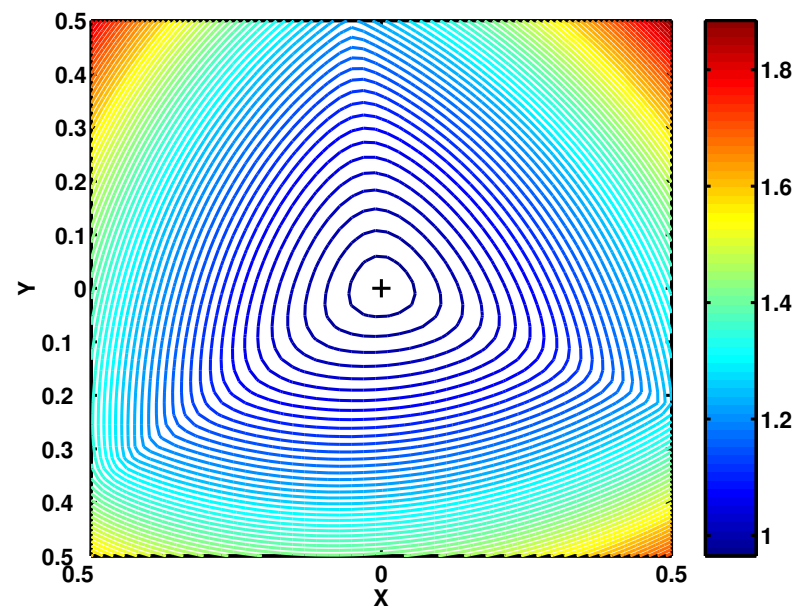

(e) $z=1.4 z_{0}$

Figure 7: Sensitivity of angular isotropy to $x, y$ in a combined isotropy configuration of the SRSPM with INRIA geometry 


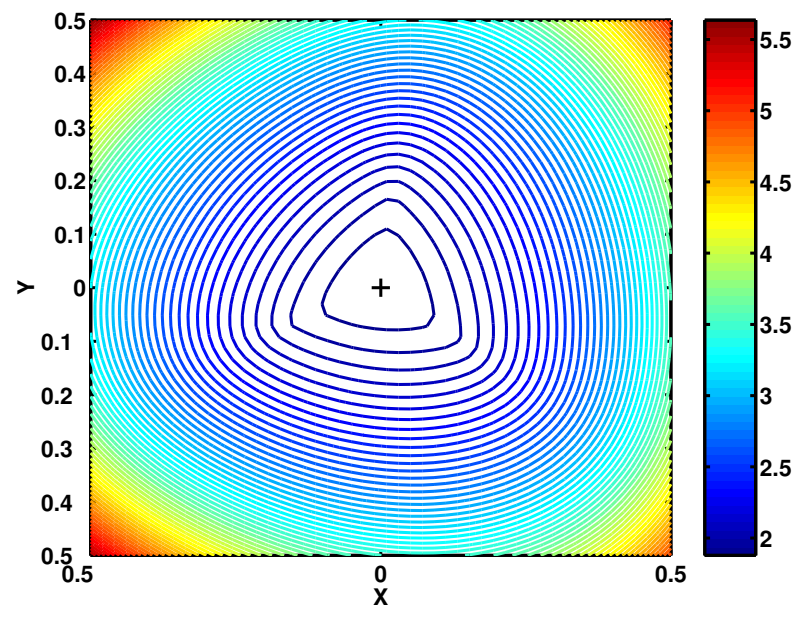

(a) $z=0.6 z_{0}$

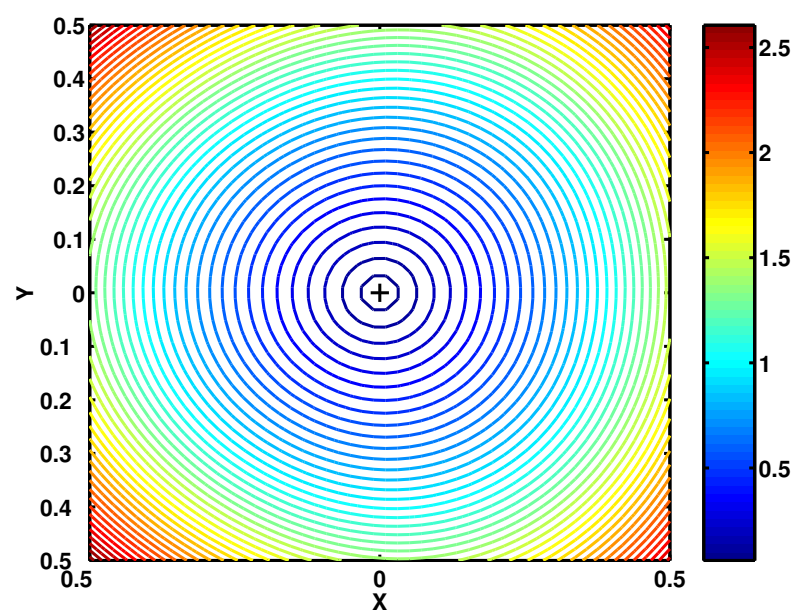

(c) $z=z_{0}$

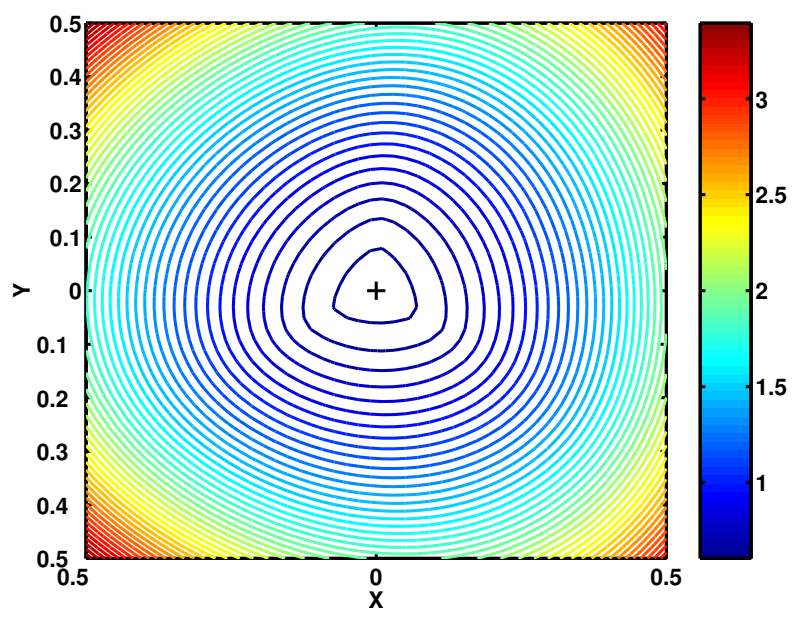

(b) $z=0.8 z_{0}$

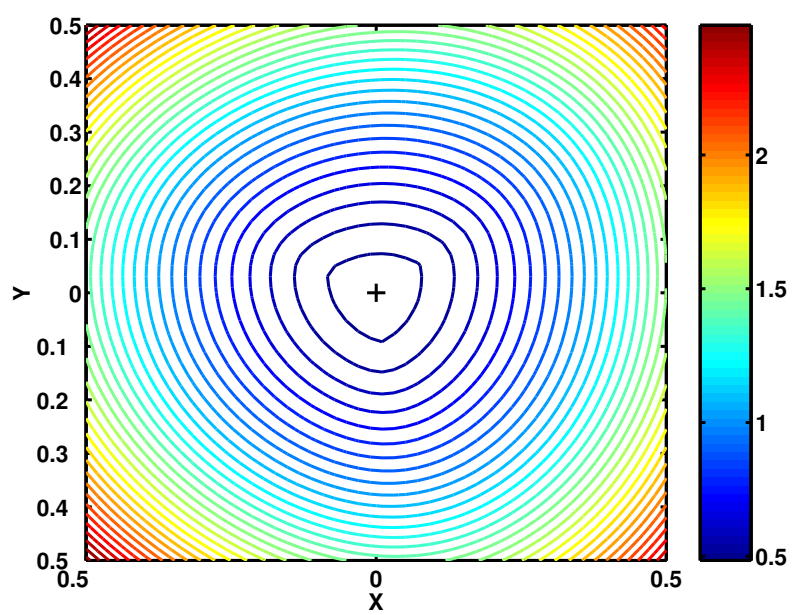

(d) $z=1.2 z_{0}$

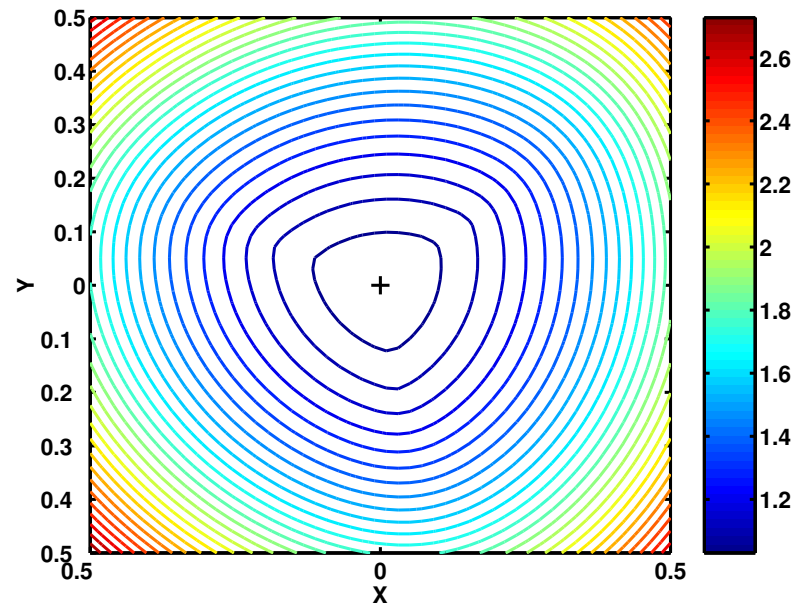

(e) $z=1.4 z_{0}$

Figure 8: Sensitivity of linear isotropy to $x, y$ in a combined isotropy configuration of the SRSPM with INRIA geometry 


\subsection{Sensitivity to $z, \phi$}

We now study a class of configurations similar to that of family 2, varying only in the $z$ location and orientation about $Z$ axis. The range of $z$ is chosen to be $\left[z_{0}-1 / 2, z_{0}+1 / 2\right]$, where $z_{0}=1.0669$ denotes the height at which isotropy is attained. The range of rotations of the top platform is chosen to be 10 degrees on either side of the isotropic orientation $\phi_{0}=-2.5097$. Figure 9 (a) shows the variation of $\delta_{\boldsymbol{\omega}}$ as a surface and figure 9(b) shows the corresponding contours. Figure 9(c), $9(\mathrm{~d})$ show the corresponding plots for $\delta_{\boldsymbol{v}}$. The '+' signs on the contours demarcate the respective isotropic configurations. It may be observed that all of these plots show prominent valleys about the

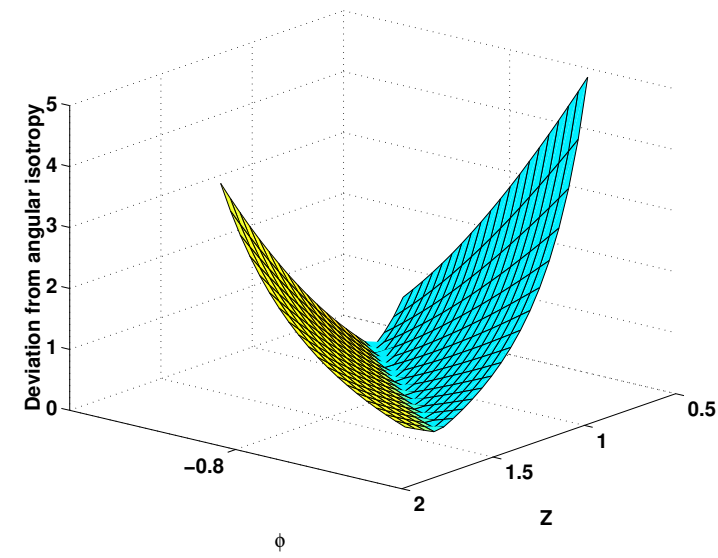

(a) Departure from angular isotropy

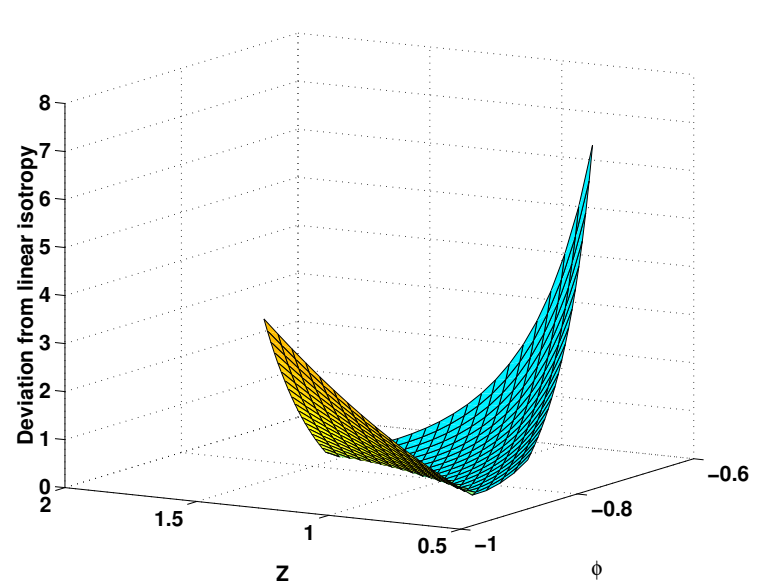

(c) Departure from linear isotropy

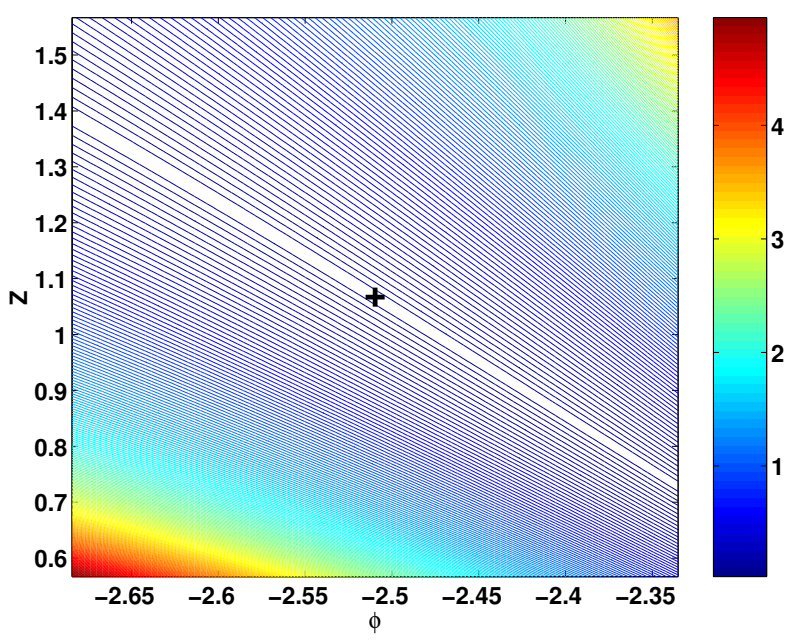

(b) Departure from angular isotropy

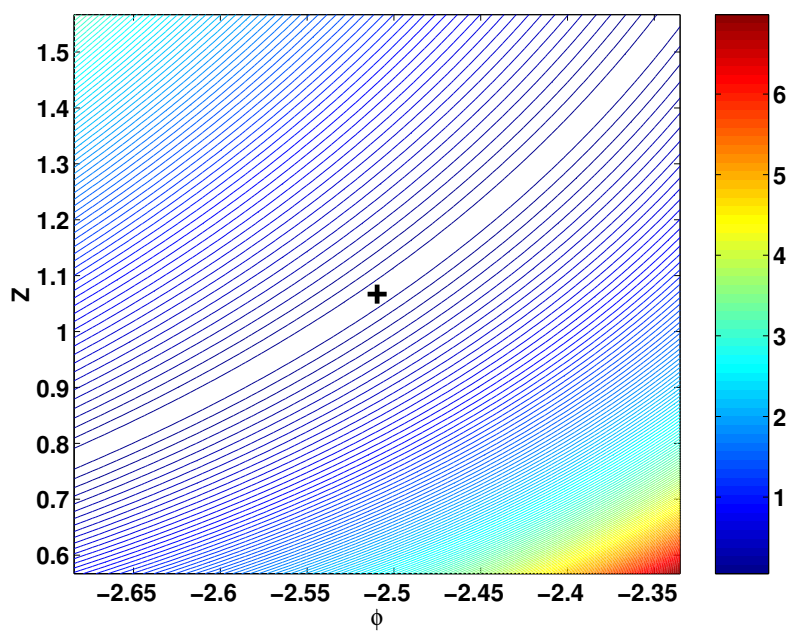

(d) Departure from linear isotropy

Figure 9: Sensitivity to $z, \phi$ at a combined isotropy configuration of the SRSPM with INRIA geometry

respective isotropic configurations, i.e., for reasonable variations of $z$ and $\phi$, the isotropy conditions 
are not disturbed much.

\subsection{Sensitivity to $\theta_{x}, \theta_{y}$}

In this case we keep the top platform position fixed, and rotate it about $X$ and $Y$ axes by $\theta_{x} \in$ $[-\pi / 18, \pi / 18]$ and $\theta_{y} \in[-\pi / 18, \pi / 18]$ respectively. In figures $10(\mathrm{a}), 10(\mathrm{~b})$, we show the contour

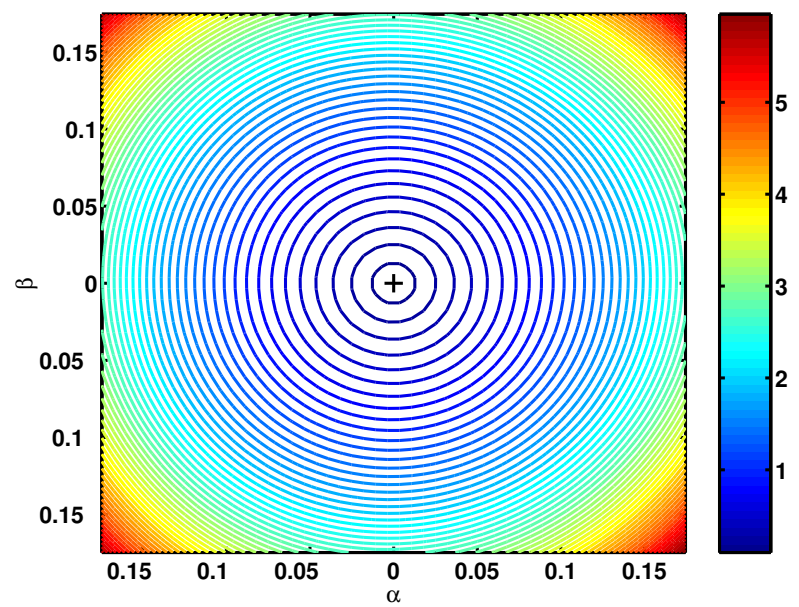

(a) Departure from angular isotropy

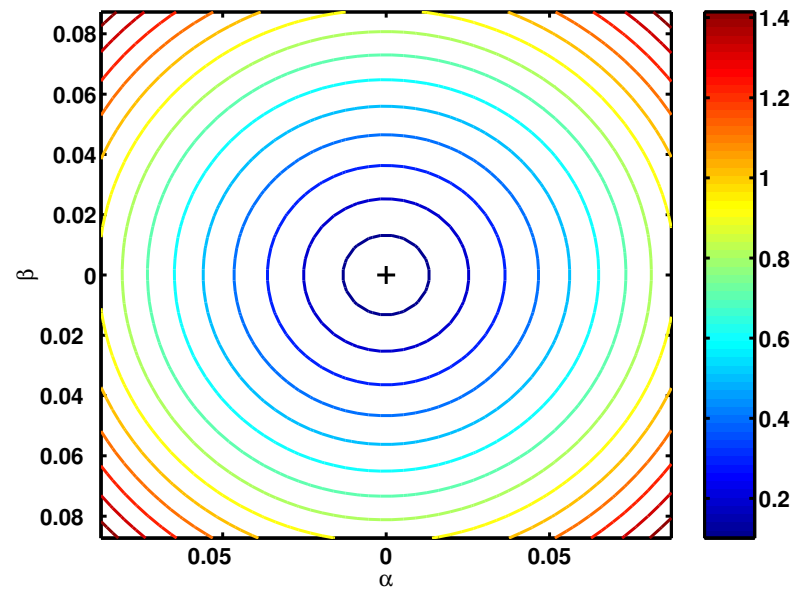

(c) Departure from angular isotropy

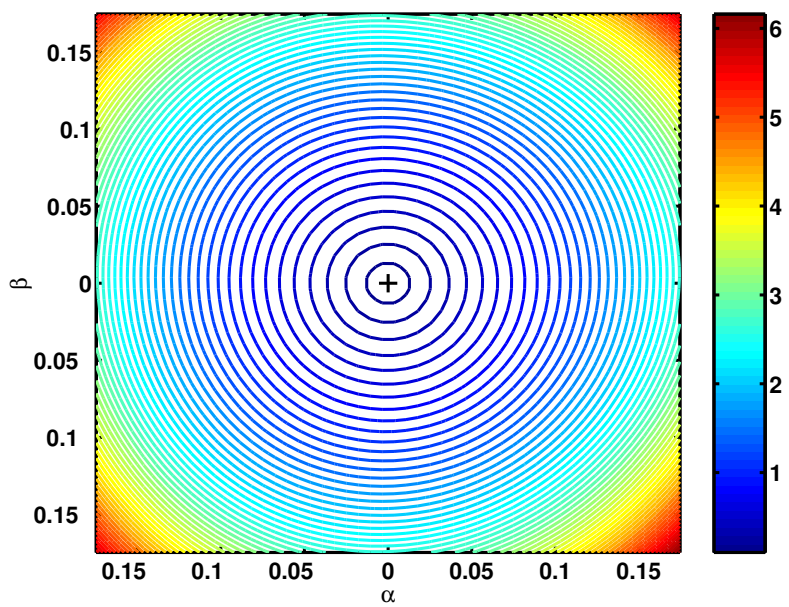

(b) Departure from linear isotropy

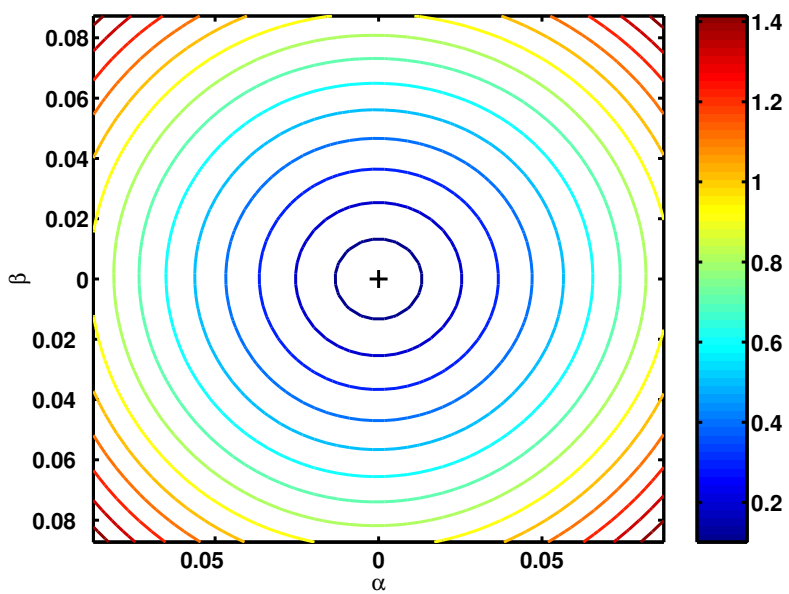

(d) Departure from linear isotropy

Figure 10: Sensitivity to $\theta_{x}, \theta_{y}$ at a combined isotropy configuration of the SRSPM with INRIA geometry

plots for deviations from angular and linear isotropy respectively. In figures 10(c), 10(d), we zoom into the portions $\theta_{x}, \theta_{y} \in[-\pi / 36, \pi / 36]$ respectively. It can be seen from these figures that the isotropic configuration at the center is reasonably insensitive to the variations of top platform orientation within the limits of 5 degrees about the $X, Y$ axis. 


\section{Conclusions}

In this paper, we have presented a formulation for studying isotropy in spatial manipulators, and applied it to a class of Stewart platform manipulators. We have derived the symbolic expressions of the Jacobian matrices corresponding to the linear and angular velocities using symbolic computation, and analysed them separately for isotropy. We have used the concept of "combined" isotropy, as opposed to spatial isotropy found commonly in literature. Using symbolic computations, we have derived two different multi-parameter families of isotropic configurations of semi-regular Stewart platform manipulators. While the first family can only show either position or orientation isotropy, the second family can show combined isotropy in a 3-parameter subspace. This family of configurations is novel in the sense that there are no established results for combined isotropy for any Stewart platform manipulator to the best of our knowledge. In addition to the analysis of combined isotropy in general, we present algorithms for the design of an SRSPM for combined isotropy at a given configuration (within family 2), and for the determination of the isotropic configurations of an SRSPM of a given geometry. We also prove that there cannot exist any configuration exhibiting spatial isotropy as defined in [9] within the two families of configurations discussed in this paper. The results are expected to help the designers in improving the dexterity of SRSPM's. Our future work includes investigating the existence of combined isotropy configurations in more general classes and/or configurations of Stewart platform manipulators.

\section{References}

[1] T. Yoshikawa, 1985, "Manipulability of robotic mechanisms," InternationalJournal of Robotics Research, 4(2), pp. 3-9.

[2] J. K. Salisbury and J. J. Craig, 1982, "Articulated hands: force control and kinematic issues," International Journal of Robotics Research, 1(1),pp. 4-7.

[3] C. A. Klein and B. E. Blaho, 1987, "Dexterity measures for the design and control of kinematically redundant manipulators," International Journal of Robotics Research, 6, pp. 72-83.

[4] K. C. Gupta and B. Roth, 1982, "Design considerations for manipulator workspace," Trans. of ASME, Journal of Mechanical Design, 104(4), pp. 704-712.

[5] R. Vijaykumar, K. J. Waldron, and M. J. Tsai, 1986, "Geometric optimization of serial chain manipulator structures for working volume and dexterity," International Journal of Robotics Research, 5, pp. 91-103.

[6] C. Gosselin and J. Angeles, 1988, "A new performance index for the kinematic optimization of robotic manipulators," Proc. 20th ASME Mechanisms Conference, Kissemmee, FL, pp. 441447.

[7] P. R. McAree, A. ESamuel, K. H. Hunt, and C. G. Gibson, 1991, "A dexterity measure for the kinematic control of a multi-finger, multi-freedom robot hand," International Journal of Robotics Research, 10, pp. 439-453. 
[8] F. C. Park and R. W. Brockett, 1994, "Kinematic dexterity of robotic manipulators," International Journal of Robotics Research, 13, pp. 1-15.

[9] C. A. Klein and T. A. Miklos, 1991, "Spatial robotic isotropy," International Journal of Robotics Research, 10, pp. 426-437.

[10] G. H. Golub and C. F. Van Loan, 1989, Matrix Computations, The Johns Hopkins University Press, Baltimore.

[11] W. A. Khan, and J. Angeles, 2006, "The kinetostatic optimization of robotic manipulators: The inverse and direct problems," Tran. of ASME, Journal of Mechanical Design, 128, pp. 168-178.

[12] M. Kirćanski, 1996, "Kinematic isotropy and optimal kinematic design of planar manipulators and a 3-DOF spatial manipulator," International Journal of Robotics Research, 15, pp. 61-77.

[13] K. H. Pittens and R. P. Podhorodeski, 1993, "A family of Stewart platforms with optimal dexterity," Journal of Robotic Systems, 10(4), pp. 463-479.

[14] K. Etemadi-Zanganeh and J. Angeles, 1997, "Kinematic isotropy and the optimum design of parallel manipulators," International Journal of Robotics Research, 16, pp. 185-197.

[15] A. Fattah and A. M. H. Ghasemi, 2002, "Isotropic design of spatial parallel manipulators," International Journal of Robotics Research, 21, pp. 811-824.

[16] K. Y. Tsai and K. D. Huang, 2003, "The design of isotropic 6-DOF parallel manipulators using isotropy generators," Mechanism and Machine Theory, 38, pp. 1199-1214.

[17] Y. X. Su, B. Y. Duan, and C. H. Zheng, 2001, "Genetic design of kinematically optimal fine tuning Stewart platform for large spherical radio telescope," Mechatronics, 11, pp. 821-835.

[18] O. Ma and J. Angeles, 1991, "Architectural singularities of platform manipulators," Proc. of the 1991 IEEE International Conference on Robotics and Automation, pp. 1542-1547.

[19] S. Bandyopadhyay and A. Ghosal, 2006, "Geometric characterization and parametric representation of the singularity manifold of a 6-6 Stewart platform manipulator," Mechanism and Machine Theory, 41, pp. 1377-1400.

[20] K. H. Hunt, 2003, "Review: Don't cross-thread the screw," Journal of Robotic Systems, 20(7), pp. 317-339.

[21] J. Angeles, 1992, "The design of isotropic manipulator architectures in the presence of redundancies," International Journal of Robotics Research, 11, pp. 196-202.

[22] R. M. Murray, Z. Li, and S. S. Sastry, 1994, A Mathematical Introduction to Robotic Manipulation, CRC Press, Boca Raton.

[23] J. Angeles, 2006, "Is there a characteristic length of a rigid-body displacement?," Mechanism and Machine Theory, 41, pp. 884-896. 
[24] Z. Wang, Z. Wang, W. Liu, and Y. Lei, 2001, "A study on workspace, boundary workspace analysis and workpiece positioning for parallel machine tools," Mechanism and Machine Theory, 36, pp. 605-622.

[25] J.-P. Merlet, 2001, Parallel Robots, Kluwer Academic Press, Dordrecht.

[26] A. Ghosal and B. Ravani, 2001, "A differential-geometric analysis of singularities of point trajectories of serial and parallel manipulators," Trans. of ASME, Journal of Mechanical Design, 123, pp. 80-89.

[27] I. N. Herstein, 1975, Topics in Algebra, John Wiley \& Sons, New York.

[28] S. Wolfram, 2004, The Mathematica Book, Cambridge University Press, Cambridge.

[29] E. F. Fichter, 1986, "A Stewart platform-based manipulator: general theory and practical construction," International Journal of Robotics Research, 5(2), pp. 157-182.

[30] D. Kim and W. Chung, 1999, "Analytic singularity equation and analysis of six-DOF parallel manipulators using local structurization method," IEEE Trans. on Robotics and Automation, 15, pp. 612-622.

[31] D. Cox, J. Little, and D. O'Shea, 1991, Ideals, Varieties, and Algorithms: An Introduction to Computational Algebraic Geometry and Commutative Algebra, Springer-Verlag, New York.

[32] R. E. Moore, 1979, Methods and Applications of Interval Analysis, SIAM Studies in Applied Mathematics, Philadelphia.

[33] J.-P. Merlet, 1999, "Determination of 6D workspaces of Gough-type parallel manipulator and comparison between different geometries," International Journal of Robotics Research, 18(9), pp. 902-916.

[34] B. M. St-Onge and C. Gosselin, 1996, "Singularity analysis and representation of spatial six-dof parallel manipulators," Recent Advances in Robot Kinematics, pp. 389-398.

\section{A Symbolic construction of the characteristic polynomial of a square matrix}

The characteristic polynomial of a square matrix $\boldsymbol{A}$ of dimension $n$ is defined as

$$
\begin{aligned}
P_{n}(\lambda) & =\operatorname{det}\left(\lambda \boldsymbol{I}_{n}-\boldsymbol{A}\right) \\
& =\lambda^{n}+a_{1} \lambda^{n-1}+\cdots+a_{n-1} \lambda+a_{n}
\end{aligned}
$$

However, construction of the polynomial from this definition requires symbolic expansion of the determinant, which is computationally very expensive, and can indeed be prohibitive. Fortunately, we can compute the coefficients $a_{i}$ above directly using a simple formula derived below from Newton's identities. 
Let $s_{k}=\sum_{i=1}^{n} \lambda_{i}^{k}, k=1, \ldots, n$, where $\lambda_{i}$ is a root of equation (59). Then for $k=1, \ldots, n$, Newton's identity states that

$$
s_{k}+a_{1} s_{k-1}+a_{2} s_{k-2}+\cdots+a_{n-1} s_{1}+k a_{k}=0, \quad k=1, \ldots, n
$$

Noting that if $\boldsymbol{A} \boldsymbol{x}=\lambda \boldsymbol{x}$, then for any positive integer $i$, we have $\boldsymbol{A}^{i} \boldsymbol{x}=\lambda^{i} \boldsymbol{x}$, and that for any square matrix the trace equals the sum of its eigenvalues, we get the relation

$$
s_{k}=\operatorname{tr}\left(\boldsymbol{A}^{k}\right), k=1, \ldots, n
$$

Finally, combining equations $(60,61)$ we get an explicit formula for $a_{k}$ as follows:

$$
a_{k}= \begin{cases}-\operatorname{tr}(\boldsymbol{A}) & k=1 \\ \frac{(-1)}{k}\left(\operatorname{tr}\left(\boldsymbol{A}^{k}\right)+\sum_{i=1}^{k-1} a_{i} \operatorname{tr}\left(\boldsymbol{A}^{k-i}\right)\right) & k=2, \ldots, n\end{cases}
$$

The above method involves only the computation of the traces of $\boldsymbol{A}^{k}$, and it is therefore much more economical than the explicit expansion of the determinant. The complexity of the algorithm can be further reduced by taking advantage of any special structure of $\boldsymbol{A}$, such as bandedness, symmetry or skew-symmetry. 